\title{
IFE Thick Liquid Wall Chamber Dynamics: Governing Mechanisms and Modeling \& Experimental Capabilities
}

A.R. Raffray ${ }^{1}$, W. Meier ${ }^{2}$, S. Abdel-Khalik ${ }^{3}$, R. Bonazza ${ }^{4}$, P. Calderoni ${ }^{5}$, C. S. Debonnel ${ }^{6}$, Z. Dragojlovic ${ }^{7}$, L. El-Guebaly ${ }^{4}$, D. Haynes ${ }^{4}$, J. Latkowski ${ }^{2}$, C. Olson ${ }^{8}$, P. F. Peterson ${ }^{6}$, S. Reyes $^{2}$, P. Sharpe ${ }^{9}$, M.S. Tillack ${ }^{7}$ and M. Zaghloul ${ }^{10}$

'Mechanical and Aerospace Engineering Department and Center for Energy Research, University of California, San Diego, EBU-II, Room 460, La Jolla, CA 92093-0417, USA, Tel: (858) 534-9720, FAX: (858) 822-2120, e-mail: raffiav@fusion.ucsd.edu.

${ }^{2}$ Lawrence Livermore National Laboratory, Livermore, CA 94550, USA

${ }^{3}$ School of Mechanical Engineering, Georgia Institute of Technology, Atlanta, GA 30332 0405, USA

${ }^{4}$ University of Wisconsin, 1500 Engineering Drive, Madison, WI 53706-1687, USA

${ }^{5}$ University of California Los Angeles, Los Angeles, CA, USA

${ }^{6}$ Department of Nuclear Engineering, University of California, Berkeley, California 94720-1730, USA

${ }^{7}$ Center for Energy Research, University of California, San Diego, La Jolla, CA $92093-$ 0417, USA

${ }^{8}$ Sandia National Laboratories, Albuquerque, NM 87185, USA

${ }^{9}$ Fusion Safety Program, EROB E-3 MS 3815, INEEL, Idaho Falls, Idaho 83415-3815, USA

${ }^{10}$ Current address: Department of Physics, College of Sciences, UAEU, P.O.B. 17551, Al-Ain, UAE. Permanent address: Department of Physics and Engineering Mathematics, College of Engineering, Zagazig University, Zagazig, Egypt.

\section{(For Submission to Fusion Science \& Technology)}

\section{December 17, 2004}

\section{ABSTRACT}

FS\&T Ms-301004 rec'd: 12/17/2004

For thick liquid wall concepts, it is important to understand the different mechanisms affecting the chamber dynamics and the state of the chamber prior to each shot as compared with requirements from the driver and target. These include ablation mechanisms, vapor transport and control, possible aerosol formation, as well as protective jet behavior. This paper was motivated by a town meeting on this subject which helped identify the major issues, assess the latest results, review the capabilities of 
existing modeling and experimental facilities with respect to addressing remaining issues, and helping guide future analysis and $R \& D$ efforts; the paper covers these exact points.

\section{INTRODUCTION}

The thick liquid wall (TLW) concept consists of a neutronically thick region of flowing liquid between the fusion target and the chamber first wall and structures. This not only provides a renewable wall to accommodate the photon and ion threat spectra from the fusion micro-explosion but also provides significant moderation and attenuation of the high energy neutron flux from the target, thereby reducing the radiation damage rate and leading to longer lifetimes for the first wall and blanket structures. By utilizing a lithiumcontaining liquid, such as the molten salts flibe $\left(\mathrm{Li}_{2} \mathrm{BeF}_{4}\right)$ or flinabe $\left(\mathrm{LiNaBeF}{ }_{4}\right)$, the thick liquid wall also serves as the tritium-breeding blanket and the primary coolant since it directly absorbs all of the short range target emissions (X-rays and target debris) and the majority of the neutron energy.

Early TLW concepts date to the 1970's [1-3]. The first detailed conceptual design work was carried out at LLNL leading to the High Yield Lithium Injection Fusion Energy (HYLIFE) chamber design [4,5]. Subsequent design modifications (including use of flibe instead of $\mathrm{Li}$ as the working fluid) led to the HYLIFE-II design [6]. The most recent integrated design based on a TLW chamber, heavy ion (HI) driver and indirect drive (ID) targets, known as the Robust Point Design (RPD), was completed in 2002 [7]. A variety of flow configurations have been proposed over the years including vortices, single and segmented annular curtains, and various combinations of rectangular and cylindrical liquid jets to make up the main TLW and create liquid beam ports.

Key issues for TLW concepts include the liquid wall behavior under the IFE threat spectra and the ensuing clearing process dictating the chamber environment prior to the next shot. This chamber environment must accommodate the target injection and driver propagation requirements. These issues were the focus of a 2003 ARIES Town Meeting on Liquid Wall Chamber Dynamics whose major objective was to bring together experts 
in these areas to identify the major issues, share the latest results, understand better the accuracy of various modeling predictions and, through discussions, to help focus future analysis and experimental $R \& D$ efforts [8]. This paper is the result of the informative presentations and discussions at the meeting and aims to provide a comprehensive summary of the status of the present understanding of these issues and of the tools available to further this understanding.

First, an example TLW chamber concept, HYLIFE-II, is presented, and the driver and target constraints summarized. Next, the mechanisms affecting chamber/liquid wall dynamics and chamber clearing are discussed. The models and experimental facilities that can be used to shed light on these mechanisms are then described. Finally, the R\&D needs are summarized and concluding remarks drawn.

\section{THICK LIQUID WALL CHAMBER CONCEPT AND OPERATION}

\subsection{Example HYLIFE-II Design with Heavy Ion Driver and Indirect-Drive Target}

Figure 1 shows a Pro/E model of the HYLIFE-II chamber as modified to be consistent with the driver and target specifications of the RPD. In particular, this latest version accommodates 120 ion beams ( 60 from each side) that are needed to drive the indirect drive target with the correct pulse shape. Figure 2 illustrates the current liquid wall configuration for the RPD in a more schematic manner. Several types of flow are required including oscillating jets to make up the central pocket, steady flow, crossing cylindrical jets in the region of beam entry, and vortices in the chamber penetrations and beam tubes leading to the final focus magnet section.

\section{INSERT FIGURE 1}

INSERT FIGURE 2 
Oscillating nozzles at the top of the chamber repetitively (6 Hz for the RPD) form a pocket of flibe (or flinabe for the RPD) around the point of the fusion target explosion at the center of the chamber. The purpose of this oscillating flow is to physically sweep any drops from the previous shot out of the central region of the chamber and provide an essentially clear inner cavity. The liquid blanket surrounding the central cavity is made up of many individual jets with an overall packing fraction of about $50 \%$. This functions as a shock absorber to reduce or even eliminate large impact stress on the first structural wall. The inner radius of the central pocket is $\sim 0.5 \mathrm{~m}$ and the liquid wall thickness is 0.56 $\mathrm{m}$ (1.12 $\mathrm{m}$ at $50 \%$ effective density). Beams enter the central cavity from two sides as shown in Fig. 1. To shield chamber structures (and focusing magnets) in the beam entry region, an array of crossing jets is used to form thick liquid beam ports. These jets should be as smooth as possible so they can be positioned close to the beams and thus provide better shielding. The injection velocity of both the central pocket jets and beam-port crossing jets is high enough $(\sim 12 \mathrm{~m} / \mathrm{s})$ to reestablish the configuration in the inter-pulse time. Vortex flow protects the beam tubes in the region between the chamber and final focus magnets. This flow can be at a lower temperature than the bulk flow to assist in vapor condensation and minimize flow up the beam lines.

From the standpoint of chamber dynamics, the key issues for the TLW design are: 1) whether the protective liquid configuration can be established to the required precision; 2) whether the liquid jet structures protect the first wall effectively; and 3) whether chamber conditions can be re-established at $\sim 6 \mathrm{~Hz}$ to allow for beam and target injection. This includes return of chamber pressure to a level that allows beam propagation and pathways essentially clear of droplets aerosols for the beams and target.

\subsection{Driver and Target Considerations}

The chamber conditions prior to each shot can impact target injection and survival as well as driver propagation. Constraints based on driver and target considerations are discussed in detail in Ref. [9] for both direct and indirect drive targets and for laser and ion beam drivers. Here, the key points are summarized. 
The temperature of the frozen DT is $\sim 18 \mathrm{~K}$ when the target leaves the injector. As the target travels to the chamber center, it is exposed to heating from energy-exchange with the chamber vapor. Direct-drive targets are very fragile and their thermal behavior during injection through the vapor is of particular concern since it could lead to unacceptable target deformation and/or density variations (when compared to target physics requirements). Indirect drive targets include a massive hohlraum surrounding the DTcontaining capsule and effectively insulating it from the heat load during the short transit to the center of the chamber. The hohlraum is illuminated from two sides by arrays of beams in a relatively narrow cone angle. These features make indirect-drive targets compatible with the TLW chamber.

There are also requirements from the driver limiting the chamber gas density. For a laser driver, laser breakdown considerations limit the vapor/gas density in the chamber [9]. For a heavy-ion driver which is the preferred option in combination with an indirect-drive target, the requirements posed on the chamber gas depend on the mode of transport and focusing [9]. Neutralized ballistic transport set the most stringent limit based on stripping with integrated line gas density equivalent to about 1 mtorr. Channel transport set the least demanding limits based on scattering with integrated line density equivalent to about 1 torr. Self-pinched transport is somewhere in between setting limits based on selfpinch resulting with integrated line density equivalent to 100 mtorr.

The example target considered for the analysis presented in this paper is a $458 \mathrm{MJ}$ heavy ion indirect-drive target [10] whose energy partition is summarized in Table I (based on LASNEX calculations [11]). The chamber liquid wall is mainly affected by X-rays and ions. Neutrons penetrate much deeper in the structure and blanket and, as such, are much less of a threat to the chamber wall. For the indirect-drive target, X-rays carry a large portion of energy (25\%) with a relatively soft spectrum dominated by soft $(<1 \mathrm{keV})$, shallowly penetrating photons, as shown in Fig.3. The photon energy deposition time is very small $(<10 \mathrm{~ns})$, which results in extremely large heat fluxes on the liquid wall giving rise to a number of ablation mechanisms, discussed in the next section. The ions carry 
less energy ( $2 \%$ for the fast ions and $4 \%$ for the debris ions), have a longer time of flight (up to $\sim \mu s$ ) than the photons and are much attenuated by the ablated material from the photon energy deposition.

INSERT TABLE I

INSERT FIGURE 3

\section{CHAMBER AND LIQUDD WALL DYNAMICS}

A number of processes affect the chamber and liquid wall dynamics at different times following the fusion micro-explosion. These are summarized in the following subsections.

\subsection{Short-Term ( 1 ms) Chamber Dynamics}

\subsubsection{Ablation Mechanisms}

As discussed in Section 2.2 , the $X$-rays carry much more energy than the ions $(-25 \%$ compared to $\sim 6 \%$ for all ions for the example indirect-drive target) and their very short energy deposition time ( $\sim \mathrm{ns}$ or less) results in large heat fluxes and liquid wall ablation. The ions carry less energy and their longer time of flight (up to $\sim \mu \mathrm{s}$ ) means that they will be significantly attenuated by the ablated material from the photon energy deposition before reaching the liquid wall. As such the ions contribute much more to raising the energy level of the already ablated material than to causing ablation itself. Consequently, the discussion of ablation mechanisms in this section focuses on the impact of the photon energy deposition on the liquid wall.

Figure 4 shows a schematic diagram of the basic physical processes and material removal mechanisms involved in the $\mathrm{X}$-ray ablation of the liquid wall. The energy deposition from the X-ray heats the skin layer of the liquid wall and produces thermal spikes. The rapid 
increase of the internal energy during these thermal spikes leads to phase change and ablation. The resulting ablation pressure pulse propagates through the liquid bulk. Reflection of the pressure pulse at a free surface or at the interface with a back wall of lower acoustic impedance than that of the liquid produces a rarefaction wave (tensile stress). If the tensile stress is greater than the strength of the material, rupture or spall occurs, establishing a new surface; this process continues until the wave is sufficiently attenuated. These processes can be illustrated through simple example computations which help to determine their relative importance for the given energy deposition regime and heating rate conditions. They are summarized below. A detailed review of these physical processes and material removal mechanisms due to photon energy deposition is presented in Ref. [12].

\section{INSERT FIGURE 4}

The major phase change (boiling) processes for a liquid subject to high energy deposition are surface vaporization, heterogeneous nucleation and homogeneous nucleation. In the case of the photon energy deposition, the heating rates are so high $\left(\sim 10^{13} \mathrm{~K} / \mathrm{s}\right.$, analogous to laser material ablation) that the surface evaporation process does not have sufficient time to occur and plays a minor role $[12,13,14]$. However, it would play a major role for heating rates corresponding to the ion energy deposition $\left(\sim 10^{9}-10^{10} \mathrm{~K} / \mathrm{s}\right)$. Similarly, results reported in Refs. [12,14] indicate that heterogeneous nucleation would also play a minor role under the extremely high photon heating rates. Instead, for such extremely high heating rates the boiling process is dominated by homogeneous nucleation, which leads to "explosive boiling". This involves rapid superheating to a metastable liquid state with a large excess free energy, which decomposes explosively into liquid and vapor phases. Under these conditions, as the temperature approaches $-90 \%$ of the critical temperature an avalanche-like explosive growth in the homogeneous nucleation rate (by 20-30 orders of magnitude) leads to this explosive boiling [14]. A discussion of more detailed estimates of explosive boiling is given in Ref. [12]. Here, for simplicity the $90 \%$ critical-temperature criterion is used to provide an example of the amount of a flibe liquid wall ablated by the photon energy deposition, as illustrated in Figure 5. 


\section{INSERT FIGURE 5}

Figure 5 shows the spatial profile of the photon energy deposition within a flibe liquid wall at a radius of $0.5 \mathrm{~m}$ from the micro-explosion. A line representing the cohesion energy (the total evaporation energy) is shown, illustrating the thickness of flibe that would be evaporated $(\sim 103 \mu \mathrm{m})$. There would be a two-phase flibe region below this threshold (the lower limiting line representing the sensible energy is not shown in the figure as it is off-scale). It is not clear to what extent this two-phase region will ablate or remain on the surface. However, it seems clear that at least the part of this region experiencing explosive boiling will ablate. Superimposing a line corresponding to $90 \%$ of the critical temperature (as suggested by Ref. [14]) shows that the explosive boiling region is $\sim 127 \mu \mathrm{m}$ in this case, resulting in a total ejected mass of $\sim 0.8 \mathrm{~kg}$ for an assumed spherical chamber. The radius of the liquid wall from the micro-explosion center is an important parameter as it determines the wall surface area seen by the photons. Increasing the liquid wall radius would result in a thinner explosive boiling region but because of the larger surface area would result in an increase in the total mass of ejected flibe. For example, the explosive boiling region thickness decreases to $\sim 10.9 \mu \mathrm{m}$ when the liquid wall radius is increased to $3.5 \mathrm{~m}$, but the total mass of ejected flibe increases to $\sim 6.2 \mathrm{~kg}$ (assuming a spherical liquid wall configuration) $[12,13]$.

The liquid wall ablation due to explosive boiling would generate an impulse creating a shock wave. Upon reaching the liquid boundary at the back of the liquid wall, this shock wave would give rise to a rarefaction wave moving inward. If the net tensile stress in the liquid due to the rarefaction wave is higher than the spall strength of the liquid, spalling would occur establishing a new liquid surface and potentially providing an additional source of ablated material. A detailed discussion of this potential ablation mechanism is given in Ref. [12] for the case of a liquid film on a solid wall where the rarefaction wave formation would depend on the liquid and reflecting wall acoustic impedances. Ref. [12] also provides a derivation of the theoretical spall strength of flibe, which is summarized in Table II. 


\section{INSERT TABLE II}

The exact shape of the pressure pulse is important in estimating the local stresses in the liquid and the possibility of spalling. Such calculations can be quite complex. Simple example estimates of spalling can be made based on the ablation pressure wave profile computed from a previous IFE study (OSIRIS [15]) with comparable X-ray yield and scaled to the case being analyzed [12]. The scaling is based on the magnitude of the relative reactive impulses (see Table III) and the pressure profile is assumed to be steady (no change in shape according to the acoustic approximation). Figure 6 shows the pressure profile from the OSIRIS study and the corresponding scaled profiles in a flibe liquid wall for the photon energy deposition from the $458 \mathrm{MJ}$ indirect-drive target. Cases with a liquid wall radius of $3.5 \mathrm{~m}$ (similar to OSIRIS) and with a liquid wall radius of 0.5 $\mathrm{m}$ (case of interest here) are both shown; for the latter case, the pressure pulse shown has been arbitrarily scaled down by a factor of $1 / 5$ so that it can be represented within the scale of the graph.

\section{INSERT TABLE III}

\section{INSERT FIGURE 6}

According to Jantzen \& Peterson [16], the peak pressure in the shock wave would decay rapidly over the first few mm's of depth; thus, for thick liquid jets, only a small fraction of the total thickness would experience high stresses. However, the theoretical spall strength of flibe is about 2 orders of magnitude lower than the magnitude of the initial shock for this case and even a "dampened" shock could result in spalling. As an illustration, a conservative estimate of spalling was made under a worst-case scenario of a steady pressure wave (i.e. no change in shape as shown in Fig. 6). The results are illustrated in Figure 7. In this case, the shock wave upon reaching the rear of the liquid wall (or curtain) gives rise to a rarefaction wave and the net tensile stress exceeds the theoretical spall strength of flibe at about $28 \mu \mathrm{m}$ from the rear of the jet, forming a new 
liquid surface.

From these simple analyses, the ablation mechanisms (such as the ejecta from explosive boiling and, possibly, the fractured liquid layer from spalling) would provide a source for aerosol formation, discussed in Section 3.1.3. The behavior of the aerosol if not swept out (e.g. by the reformation of the liquid jets) could affect the heavy ion driver focusing. Clearly, further effort are required in understanding better the complex dynamic processes associated with these ablation mechanisms through a combination of modeling and scaled experiments (simulating prototypical conditions).

\section{INSERT FIGURE 7}

\subsubsection{Isochoric Nuclear Heating}

Over the past three decades, numerous heavy ion beam (HIB) designs have been developed, employing thin and thick liquid walls to protect the solid structure against the target $\mathrm{X}$-rays and debris and therefore extend the service lifetime and improve the reliability of the structural components. The thin liquid walls tend to protect the FW but do not appreciably change the level of radiation damage or the lifetime of the structure. The intent of the thick liquid metal or molten salt systems would be to protect the chamber structure such that it would last for the life of the plant [17].

To understand the isochoric heating problem, it is essential to identify the sequence of events as well as the evolution of the liquid wall with time following the target implosion. The target threat to the liquid surface is in the form of highly energetic X-rays, neutrons, and debris ions. At the liquid surface, the neutron heating is approximately five orders of magnitude lower than the X-ray and ion heating, meaning the surface effect of the neutrons can be neglected.

The geometry of the bulk liquid hardly changes before the arrival of the neutrons. The neutrons deposit their energy volumetrically in the remaining liquid bulk (and any 
underlying structure). The neutron heating causes pressurization of the liquid. If the energy density is high enough, the liquid jets can break-up. The disassembly of the liquid wall/jets is allowed in free-jet systems, like the HYLIFE-II thick liquid wall design [6]. In the HIBALL thin liquid wall design [18], the pressure is lower because of the much larger surface radius $(\sim 5 \mathrm{~m})$ and the liquid is contained in thin porous tubes.

The physical geometry of the two representative designs (HYLIFE-II and HIBALL) and the flow of the protecting liquids are quite different. Although the total energy assumed in both studies is around $400 \mathrm{MJ}$, HYLIFE-II has a much smaller radius $(0.5 \mathrm{~m})$ from the target to the liquid wall as compared to $5 \mathrm{~m}$ for HIBALL. The time-integrated power density deposited in the wall is much larger for the HYLIFE-type designs $\left(\sim 150 \mathrm{~W} / \mathrm{cm}^{3}\right)$ as compared to $2 \mathrm{~W} / \mathrm{cm}^{3}$ for HIBALL. The time integrated power density in both designs decreases roughly by $2 \%$ within the first one $\mathrm{cm}$ of the liquid, unlike the X-ray and ion energy deposition that diminishes in a few microns.

Due to the pulsed nature of IFE systems, it is instructive to examine the results of timedependent nuclear analyses. The IFE fusion reactions occur during a very short burn time (10-100 ps). Most of the high-energy neutrons reach the liquid surface in 10-150 ns, depending on the surface radius. The lower energy neutrons arrive over a longer period of time. The neutrons spend tens of nanoseconds slowing down within the liquid blanket. The HIBALL study performed rigorous time-dependent heating analysis for both liquid and structure of an IFE power plant [19]. A more recent time-dependent study [20] has focused on the neutron flux within $\mathrm{Fe}$ and $\mathrm{SiC}$ structures and the neutron-induced radiation damage for thick flibe and $\mathrm{Pb}-17 \mathrm{Li}$ liquid wall systems with no evaluation for the isochoric nuclear heating.

The variation of power density with time at the liquid surface of HIBALL is shown in Fig. 8 [19]. From the figure, the following broad trends emerge: (1) the peak is approximately $10^{7}$ times the average based on the chamber rep-rate; (2) the temporal distribution has a narrow width of $\sim 20 \mathrm{~ns}$; and (3) the heating diminishes in 
microseconds. Results such as these provide input to calculations of liquid response to neutron heating.

\section{INSERT FIGURE 8}

\subsubsection{Shock Propagation through Chamber}

Due to the arrangement of the numerous liquid jets, shock propagation through HYLIFEII-type chambers is far more complex than in dry-wall or thin-liquid IFE chambers. In a thick-liquid chamber, indirect-drive target X-rays ablate a thin layer off the surface of the inner pocket; fast ions quickly deposit their energies in the ablated gas; and slow target debris interacts with the expanding ablated layer. A complex pattern of reflected and transmitted waves is then generated, in which waves can be transmitted through the jet structures, or reflected off the inner liquid pocket or other waves. The gas will ultimately vent through the various thick-liquid jets, filling in the volume of the target chamber.

In the HYLIFE study, it was realized early on that a closed pocket (beside leaving no room for target or driver propagation) would result in an excessive pressurization of the inside pocket and the liquid curtain being slammed into the structural first wall. Both HYLIFE-II and the RPD rely on oscillating slab jets with venting openings to avoid pocket over-pressurization. HYLIFE-II makes use of a "slot pocket," made of a row of distinct oscillating slab jets and ablated flibe vents through the slots. The RPD uses a "hybrid" configuration with large voided slab jets for better shock abatement and a large venting opening to favor rapid target and ablated debris venting towards the liquid droplet spray on the sides of the chamber. The propagation of the blast wave inside the jets, over longer times scales, is described in Section 3.2.3. Assessment of the efficiency of the venting process is essential and has been carried out with the gas dynamics code TSUNAMI [21,22], to be described in Section 4.1.3. Results from gas dynamics simulations set the initial conditions for the pocket response to the ablation and pocket pressurization impulse load. The pressure exerted on the structural first wall by the gas can also be investigated $[23,24]$. 
Another crucial issue is the propagation of target and ablated gas up the beam lines where it could deposit and cause arcing between the still un-neutralized beam and the tube wall. Although pockets can be designed to maximize gas venting in directions opposite to the beam lines, TSUNAMI helped recognized that this approach would not be effective enough. As mechanical shutters are too slow to close off the beam lines quickly enough, a cold flinabe liquid vortex layer was suggested to coat and protect the beam line near the target chamber [21], but it was realized it could not be long enough for all the debris and ablated gas to condense [25]. The combination of an ionizing plasma and a weak magnetic dipole was proposed to effectively stop the debris and prevent their ingression past the vortex [25]. The dipole will prevent the neutralizing electrons from streaming up the beam line as well, hence limiting beam emittance growth. However, further work is required to fine-tune these "magnetic shutters." Fig. 9 shows the RPD beam line schematic.

\section{INSERT FIGURE 9}

State-of-the-art two-dimensional gas dynamics simulations of the RPD chamber are presented in Section 4.1.3. Advancement in simulating gas dynamics in thick-liquid IFE systems is currently underway with the development of multi-dimensional, multi-species, multi-phase gas transport models [see for instance [26]).

\subsubsection{In-Flight Aerosol Formation}

Material vaporized from the thick protective wall following an IFE micro-explosion could at some time be in a state suitable for particulate condensation in addition to film condensation onto the thick-liquid walls or droplet spray. Aerosol nucleation and growth likely occur as sufficient material cools during expansion from the ablated region (as understood from gas dynamics simulations discussed in Section 4.1.5), leading to what is termed in-flight aerosol formation within the chamber. Particulate growth and behavior are described by the aerosol dynamic equation [27], which is coupled to the gas dynamic 
equations by source/sink terms in mass, energy, and momentum. The aerosol dynamic equation balances the contribution of various mechanisms in the change of the aerosol population. Transport mechanisms such as convection, diffusion, and external forcing (e.g. gravitational and electro-dynamic forces) are part of the equation, as are terms representing rates of change in the aerosol size distribution due to coagulation and particulate growth (homogeneous nucleation and condensation growth). For the purpose of simulating IFE-relevant conditions, diffusion and deposition are generally not considered since they impact an aerosol population on time scales larger than the typical IFE inter-shot frequency. Other phenomena that are important in IFE chamber dynamics, for example ion-induced nucleation, splashing of melt at the surface, and aerosol impact deposition and reflection, are being modeled to extend the usefulness of the simulations. It is important to note that although flibe is a leading candidate material for the thickliquid wall protection scheme, present modeling capabilities for gas and aerosol behavior are incomplete for the simulation of this material. Example results illustrating these mechanisms presented here are therefore provided for a single component material, namely liquid lead.

In-flight aerosol formation is possible since very high nucleation and growth rates are found at chamber locations with significant supersaturation caused by rapid expansion cooling of vaporized wall material. Scoping studies for possible wall materials in IFE systems have shown aerosol particles may be formed by homogeneous nucleation for time periods shortly after the vaporized material begins to cool. Figure 10 illustrates the results for liquid lead. Part (a) of the figure shows homogeneous nucleation rates and droplet critical radii (the size at which nucleated particles have stable growth rate) as saturation ratio increase for various vapor temperatures. A vapor that cools to $1500 \mathrm{~K}$ (from an estimated 7000-8000 K ablative layer) forms $0.8 \mathrm{~nm}$ particles at a rate of $10^{20}$ particles $/ \mathrm{m}^{3} / \mathrm{s}$ at a saturation ratio of 5 . Particles of this size are composed of about 5 atoms of neutral lead. Nucleation rates are a strong function of saturation ratio; doubling the saturation ratio from 5 to 10 increases the rate by 6 orders for a vapor at $1500 \mathrm{~K}$. Part (b) of the figure gives the time required to nucleate $10^{15}$ particles $/ \mathrm{m}^{3}$, a concentration at which coagulation becomes increasingly important in the IFE chamber. For the 
conditions mentioned above, the time required to nucleate particles to this concentration is $\sim 80 \mu \mathrm{s}$, demonstrating that in-flight aerosol formation is a relevant mechanism for early-time chamber dynamics.

\section{NSERT FIGURE 10}

Aerosol particles that are formed from homogeneous nucleation may continue to grow due to surface vapor deposition in the presence of vapor that is not depleted by nucleation alone. This mechanism, termed condensation growth, is also a function of vapor saturation ratio, albeit to a much lesser extent than homogeneous nucleation. Figure 11 shows the time needed for a $10 \%$ increase in volume of a $1 \mathrm{~nm}$ lead particle as a result of condensation growth. This volume increase reflects a significant change in the aerosol population size distribution, which in turn impacts aerosol growth and transport behavior. With the particle and vapor temperature at $1500 \mathrm{~K}$, the required time for $10 \%$ volume growth is less than $1 \mu \mathrm{s}$ for all saturation ratios greater than 5 . Condensation growth rapidly slows when less vapor is available, i.e. the saturation ratio approaches unity. In a real condensing system, both homogeneous nucleation and condensation growth may occur to varying degrees during the same time period once some population of particles have formed, and their competing rates are coupled through the amount and state of available vapor.

\section{INSERT FIGURE 11}

\subsection{Intermediate-Term ( $100 \mathrm{~ms})$ Chamber Dynamics}

\subsubsection{Film Condensation on Cold Surfaces}

The net film condensation can be expressed by the difference between the condensation flux to the liquid surface and the evaporation flux from the liquid surface. In Ref. [13], a characteristic condensation time based on condensation rate and corresponding vapor mass in the chamber is used to estimate the time required for film condensation to clear 
the chamber as a function of vapor pressure and temperature for both $\mathrm{Pb}$ and flibe. The results for an example $5 \mathrm{~m}$ chamber indicate that, for a given vapor temperature, the characteristic condensation time is virtually independent of the vapor pressure until it decreases to within about one order of magnitude of the saturation pressure corresponding to the liquid film temperature. This characteristic time $(<0.04 \mathrm{~s})$ is significantly smaller than the time between shots $(0.1-1 \mathrm{~s})$ showing that condensation itself is fast. The overall film condensation process in a chamber would probably be more limited by vapor transport to the liquid surface. However, the vapor pressure prior to each shot will be higher than the liquid saturation pressure by up to a factor of $\sim 10$ (as reference, for $\mathrm{Pb}$ at $1000 \mathrm{~K}$, the saturation pressure is $\sim 1.1 \mathrm{~Pa}$; and for flibe at $800 \mathrm{~K}$, the saturation pressure $\sim 0.0063 \mathrm{~Pa}$ ). To alleviate this concern, a spray of droplets colder than the liquid jet structures can be employed. The combination of surface renewal (by the continuous introduction of fresh droplets) and low temperature of the injected droplets enhances condensation and helps in a faster attainment of vapor pressure equilibrium.

\subsubsection{Aerosol Coagulation and Evolution}

Coagulation describes the process of two aerosol particles (or droplets) colliding to become one particle with a volume equal to the sum of the volumes of the initial particles without affecting general particle shape. Aerosols produced by in-flight nucleation and growth within the chamber will experience collisions and coagulation at time periods beyond that of formation. Unlike nucleation and growth mechanisms, coagulation is comparatively less dependent on fluid state properties, but is itself dependent on the size distribution. Figure 12 illustrates the time characteristics of coagulation by examining the time required for changing a given number density of $1 \mu \mathrm{m}$ aerosol particles. The closeness of the curves representing different temperatures illustrates the weak dependence on the fluid state. An initial concentration of $10^{15}$ particles $/ \mathrm{m}^{3}$ at $1 \mu \mathrm{m}$ in size requires $100 \mathrm{~ms}$ to alter the size distribution by increasing average particle size while reducing the total number (in a unit volume) by $10 \%$. This simplified analysis shows that coagulation should be considered as a longer-term chamber dynamics transport mechanism. It also gives some credibility to the assumption that gravitational settling 
and removal of the aerosol particles is unlikely during the inter-shot period. Large particles $(>\sim 50 \mu \mathrm{m})$ are necessary for gravitational settling to become important, and the analysis shows that $1 \mu \mathrm{m}$ particles cannot grow to an average size of $50 \mu \mathrm{m}$ within $100 \mathrm{~ms}$ [27]. In the case of HYLIFE-II, estimates of aerosol population shortly before the next shot should take into account the effect of the "larger" droplet spray, which would substantially enhance surface vapor condensation and reduce the chamber vapor pressure, thereby mitigating the aerosol concern.

\section{INSERT FIGURE 12}

\subsubsection{Jet Reformation}

\section{Jet Structures}

In the original HYLIFE-II design, the protection of the first wall and final-focus magnets was provided by an array of slab jets and an oscillating liquid pocket. The main ideas have been retained for the RPD target chamber, with a few modifications [28]. "Voided" slab jets are used for the pocket, as they provide a better abatement of shocks. Voided slab jets are made of a sheet jet and an array of packed cylindrical jets.

The use of cylindrical jets is recommended to protect the beam ports. A "vortex" is used to coat the last few meters of the beam tube; it serves as a buffer between the target chamber and final-focus magnet region where the requirements on background gas and cleanliness are different. The background gas blowing from the target chamber is expected to condense on the cold vortex before reaching the final-focus magnet region. Oscillating voided slab jets, cylindrical jets and vortex flows have been demonstrated to have the required geometric precision in scaled experiments [28].

The corrosion-induced weariness of the nozzles and their possible obstruction by target debris still need further analysis. Molten salts corrosion and purity can be controlled effectively to limit corrosion and debris concentration through careful control of redox 
potential and constant purification of the coolant. Purification techniques depend on the choice of the target and hohlraum materials; definitive work on this issue will require a flibe recirculation loop.

\section{Pocket Disruption}

Studies of disruption of voided slab jets have been conducted at the University of California at Berkeley. The snowplow model of shock propagation through a voided jet structure has been successfully developed and benchmarked against experimental data. As the shock propagates through the voided jet, the effective liquid density increases, as if a "molten-salt-plow" was crushing the cylindrical jets. The process is illustrated in Figure 13. Use of jet structures with a $50 \%$ packing fraction ensures the pocket is already in the pool at the bottom of the chamber before the shock wave has time to reach the back of the pocket; this is essential to prove that no high-speed droplets are ejected from the back of the liquid pocket towards the structural first wall.

\section{INSERT FIGURE 13}

Timely recovery of the oscillating pocket has been demonstrated experimentally [28]. Shock waves traveling upward could disrupt the jets before they form the next pocket at the center of the chamber, or might even damage the nozzles. This set restriction on the range of pocket shapes that can be employed [29]. Studies of the disruption of the array of cylindrical jets have yet to be performed but a similar disruption model is expected to apply.

\section{Hydrodynamic Droplet Source Term}

The "hydrodynamic droplet source term" refers to droplet production by primary turbulent breakup of liquid jets. Recent publications in this area suggest that liquid jets in the regimes of interest to thick liquid protection concepts may be inherently unstable and susceptible to primary turbulent breakup $[30,31]$, whereby droplets are continuously 
ejected from the surface of the jets and spread about the chamber, possibly interfering with driver propagation and target delivery. Empirical correlations have been reported for the onset of primary breakup, Sauter mean diameter of the ejected droplets, and droplets' mass flux for turbulent, unconditioned, annular and round jets with exit conditions corresponding to fully-developed channel flow [31]. Application of such correlations to unconditioned round jets with dimensions like the jets used in the stationary protective lattice of the RPD-2002 design predicted a large hydrodynamic source term $[28,32]$.

Based on these results, an experimental investigation has been undertaken to determine whether flow conditioning and/or boundary layer cutting can reduce the hydrodynamic source term to a sufficiently low level compatible with beam propagation requirements [32]. Vertical turbulent sheets of water at near prototypical Reynolds number $\left(1.3 \times 10^{5}\right)$ issuing downward from nozzles with exit cross sections of $(1 \times 10 \mathrm{~cm})$ were examined. A simple mass collection technique was used to measure the rate of droplet ejection from the jet surface at different locations along the flow direction. Several flow conditioning schemes were examined to establish the relative importance of traditional flow straightening elements. The effect of boundary layer cutting on the hydrodynamic source term was also quantified [32]. The results indicate that standard flow conditioning schemes in combination with contracting nozzle designs can reduce the droplet mass flux from turbulent breakup by $3-5$ orders of magnitude, and that boundary layer cutting in conjunction with standard flow conditioning can eliminate the hydrodynamic droplet source term, provided that fine-mesh screens are included in the flow conditioning elements [32]. For these reasons, conditioning and boundary layer trimming are used in the RPD-2002.

\section{MODELS AND EXPERIMENTS}

Understanding and characterization of the different mechanisms affecting the liquid-wall chamber dynamics (described in the previous section) are very important in designing the 
chamber and in being able to estimate key parameters such as the aerosol concentrations in beam lines prior to each shot (which must be compatible with the driver requirements). Models and experimental capabilities that can simulate IFE conditions are key tools in succeeding in this endeavor. Many such models and experimental facilities already exist and might only need specific modifications to address these issues. A list (non-exclusive) of the known models and experimental facilities available in the US is shown in Table IV in terms of their applicability to address issues linked with liquid wall chamber mechanisms occurring at different times following the fusion micro-explosion. They are described in the following subsections with a view of helping to better recognize where there are gaps in current understanding and capabilities and where future $R \& D$ effort should be directed. This represents just a starting point for a process which must be much more thorough (such as, for example, considering the possibility and cost of adding new capabilities to experiments and of running the experiments) to arrive at a clearer vision of a future $R \& D$ plan based on a given budget.

\subsection{Models}

Existing numerical models described here are: BUCKY developed at the University of Wisconsin (UW), Madison; ABLATOR developed at the Lawrence Livermore National Laboratory (LLNL) and the University of California at Berkeley (UCB); TSUNAMI developed at UCB; SPARTAN developed at the University of California, San Diego (UCSD); and TOPGUN developed at the Idaho National Laboratory (INL). This list is not exclusive as there may be other models that could be adapted to help address some of the key issues associated with liquid wall chamber dynamics. However, they provide a good snapshot of the capabilities of current modeling tools and of their possible application to help solve those issues.

\subsubsection{BUCKY}

BUCKY, a one-dimensional Lagrangian radiative-hydrodynamics code [33], is used to simulate the response of the chamber gas and wall to target $\mathrm{X}$-ray and ion threat spectra. 
Prompt X-ray deposition is modeled using cold opacities from Biggs and Lighthill. Deposition of ion energy is approximated by the theory of Melhorn [34] and the free electron contribution interpolates between the low energy Lindhard-Scharff limit and the high energy Bethe limit. Radiation transport [35] is calculated in the flux-limited multigroup diffusion approximation. Energy that reaches the wall is treated as a source term in a thermal diffusion equation. As the temperature in a wall cell approaches the vaporization temperature, the zone begins to vaporize at a rate determined by the relative rates of vaporization and condensation, as determined by the kinetic theory of Labuntsov and Kryukov [36.

BUCKY has been originally developed for analysis of dry wall chambers with minimal wall ablation. For a liquid wall, the relevance of a one-dimensional simulation of chamber response past the time when the vapor ejected from the wall meets the shock of the chamber vapor pushed outward from target chamber center is questionable. The code simulations would also lead to an optimistic chamber response scenario since the code does not include the effects of aerosolization and possible liquid injection into the chamber (for example, due to explosive boiling or scattered droplet formation from jet instabilities). BUCKY has been used to simulate the response of a thin $\mathrm{Pb}$ liquid wall chamber to the threat from the $458 \mathrm{MJ}$ heavy ion indirect drive target. The results indicate that the dense ionized vapor formed by the interaction of the early part of the $\mathrm{X}$ ray pulse shields the surface from later $\mathrm{X}$-rays and ions, reradiating the absorbed energy in time scales long compared to the pulse from the target [13]. These results tend to be conservative given the code's limitations when applied to a liquid wall but they provided a lower bound estimate of ablated material for aerosol calculations. BUCKY would need to be substantially upgraded to correctly simulate the response of a thick liquid wall chamber configuration such as HYLIFE-II, including more comprehensive modeling of the shock wave in the chamber, inclusion of the effect of different ablation source terms and of in-flight condensation and aerosolization, and better simulation of a multidimensional geometry.

\subsubsection{ABLATOR}


ABLATOR ("Ablation By LAgrangian Transient One-dimensional Response") is a 1-D finite difference code for the calculation of material response to $\mathrm{X}$-rays that has been. developed at the Lawrence Livermore National laboratory and the University of California at Berkeley [37]. The code uses an explicit scheme for advancing in time (conditions at the next time step are calculated directly from the state at the current time step plus any incremental energy input). Four processes are modeled: energy deposition from the $\mathrm{X}$-rays, transient thermal conduction, thermal expansion (which raises pressures and causes hydrodynamic motion), and removal of material through surface vaporization and various spall processes.

LLNL has recently updated and debugged the ABLATOR code in order to generate an enhanced version for use in IFE. The most relevant modifications include: (1) implementation of direct and indirect drive X-ray spectra; (2) ability to account for attenuation through a background gas; (3) introduction of a restart capability; (4) generation of a multi-material version of the code; and (5) addition of new materials (W and flibe) to the code's material database.

In order to assess the use of ABLATOR for the special case of IFE liquid walls, a series of runs were carried out to compare the ABLATOR results against those from the TSUNAMI code described in Section 4.1.3. For this purpose, a 40 ns pulse from a single energy line of $113 \mathrm{eV}$ was assumed (based on XAPPER parameters), and results were obtained on flibe ablation depth for a series of different $X$-ray fluences. The results of these calculations showed a very good agreement between the two codes (see Table V). It was found that ABLATOR's vaporized depths were slightly smaller than those calculated with TSUNAMI. This result is consistent with the fact that ABLATOR considers heat conduction during the pulse, whereas the energy deposition is instantaneous in TSUNAMI.

\section{INSERT TABLE V}


LLNL also estimated the flibe ablation thickness under the real HIF spectrum for a flibe pocket at $0.5 \mathrm{~m}$ from the target, and for a thin film at different distances from target. The results yielded a total initial ablated thickness of $150 \mu \mathrm{m}$ in the case of the thick liquid pocket. Figure 14 shows the results for the case of a thin flibe film as a function of distance from the target. It can be observed that in the case of a wetted wall at $6.5 \mathrm{~m}$, the estimated initial ablated thickness is $2.2 \mu \mathrm{m}$.

\section{INSERT FIGURE 14}

Finally, it must be pointed out that the ABLATOR code was originally developed for modeling ablation of the National Ignition Facility dry wall under low fluences where, in the absence of plasma formation, the assumption of cold opacities is adequate to model photon-matter interaction. The main limitations of the use of ABLATOR for IFE liquid chambers are based on the lack of models for hot opacities, re-radiation and condensation. The use of cold opacities in the code assumes that the attenuation of photons at a given energy level stays constant throughout the run. However, if a plasma is generated during $\mathrm{X}$-ray deposition, this cold-opacity assumption would no longer be valid. The results presented here are a mere estimation of initial ablated mass caused by the arrival of the X-rays. For a more realistic simulation of the ablation of flibe under IFE $\mathrm{X}$-ray irradiation, it is recommended to consider models that account for additional phenomena such as re-radiation from hot vapor, surface condensation and evaporation of the ablated material, and use of hot opacities.

\subsubsection{TSUNAMI}

TSUNAMI refers to a series of hydrodynamics codes developed and maintained since the early 1990's at the University of California at Berkeley (UCB). The first two versions of the code were developed to model the gas dynamics inside the original HYLIFE-II target chamber [6]. Chen [38] developed the first one-dimensional (1-D) version to assess Xray ablation and hydrodynamics expansion of target debris and ablated molten salt in the interior of the thick-liquid pocket. Concurrently, Liu [23,24] wrote the first two- 
dimensional (2-D) version of TSUNAMI to model hydrodynamics venting through the "slot" array of slab jets. In addition, Liu [23] developed a 1-D version that included a condensation/evaporation model. TSUNAMI was later modified and employed to model ablation and gas dynamics phenomena in the National Ignition Facility (NIF) target chamber $[39,40]$. Later on, Scott's version was expanded to include some radiation transport [41] and assess its effects on the gas dynamics in the HYLIFE-II chamber. Simulations encompassing both the inside of the target chamber and the array of jets were presented in Ref. [41].

TSUNAMI was then upgraded with a user-friendly input file builder and output file processor. This version, TSUNAMI 2.8, was used to predict the mass and energy fluxes at the beam ports of a HYLIFE-II-like chamber [21]. TSUNAMI 2.8 was then employed to model the gas dynamics inside a beam tube [25] and the first "integrated" simulation was presented in Ref. [22]. This simulation covered the whole domain of early-time gas dynamics, namely from the target explosion location to the site of the magnetic shutters. Figure 15 shows snapshots of the gas density at various times in the RPD chamber [22]. The simulation shows how the target and ablated debris pressurize the pocket and then vent through the thick-liquid structures.

\section{INSERT FIGURE 15}

The TSUNAMI physical models and assumptions used for the RPD simulations are summarized below.

- The compressible Euler equations are solved through a Godunov's scheme.

- Viscous and dissipative effects are neglected since viscous and dissipative time scales are much longer than the typical run time of order of a millisecond.

- A real gas equation of state is used.

- Mass transfer is neglected and the composition of the ablated molten salt is assumed to remain stoichiometric.

- Radiation transport is neglected since earlier simulations including radiation transport showed that radiation plays a secondary role. 
- Initial conditions are given by considering the relevant phenomena in the very short term (such as photon and ion energy deposition).

- Neutrons are neglected since the background gas and ablated molten salt are virtually transparent to the neutrons. The neutron energy will be deposited much deeper in liquid structures. This would cause isochoric heating and could induce disruption of some jets. Assuming that proper design of the target chamber would avoid generation of high-speed slugs $(>1 \mathrm{~m} / \mathrm{s})$, neutron isochoric heating can be neglected for submillisecond simulations.

- A fairly efficient model computes the ablation thickness and the energy profile of the $\mathrm{X}$-ray ablated layer.

- Fast ions will be stopped in the expanding ablated molten salt. The mass of fast ions is small compared to that of ablated molten salt, and their energy is a small compared to the target energy converted into X-rays (2\% vs $25 \%$ ). Their effect is neglected in the RPD simulation. Slow target-debris ions are modeled as a sphere of molten salt.

- Two different boundary conditions are imposed: open and reflective. An open boundary is used whenever possible to limit the size of the computational domain. A reflective boundary simulates a solid surface or an axis/plane of symmetry. Most versions of TSUNAMI assume that a liquid boundary could be modeled as a reflective, stationary boundary.

- Convective transport is assumed to dominate heat and mass transfer in the vicinity of the jets, heated up to high temperatures by the target $\mathrm{X}$-rays.

- The liquid vortex surface is assumed to be perfectly condensing, due to its low temperature. This assumption was useful to show the necessity of using magnetic shutters to supplement the annular vortex. The droplet spray is assumed to be perfectly condensing as well.

TSUNAMI 2.8 was recently employed to model a variant of the thick-liquid RPD chamber that accommodates the assisted-pinch final transport scheme [42]. TSUNAMI 2.8 also showed good simulation of the gas dynamics phase from LLNL's Condensation Debris Experiment [43]. UCLA, in collaboration with UCB, has implemented Liu's model into an early version of TSUNAMI 2.8 and used the code to model UCLA flibe 
condensation experiment, described in Section 4.2.2 [44]. Agreement between simulation and experimental results was satisfactory.

The next version of TSUNAMI, "Visual Tsunami" makes use of modern programming languages and software, and includes a user-friendly input file builder and output file processor [26]. It includes a three-dimensional, multi-species, ablation and hydrodynamics core with condensation boundaries based on Schrage's model [45]. Visual Tsunami is being benchmarked. Models for radiation and aerosol transport have been developed and may be implemented into future versions of Visual Tsunami [43].

\subsubsection{SPARTAN}

SPARTAN is being developed at UCSD as a fully integrated computer code for modeling and studying dry-wall chamber dynamic behavior in the hydrodynamics time scale, including: the dynamic gas response to target implosion, the effects of various heat sources and transfer mechanisms such as photon and ion heat deposition and chamber gas conduction convection and radiation; the chamber wall response and lifetime and the cavity clearing. At present, SPARTAN solves the 2-D transient compressible NavierStokes equations. The code is written in a modular fashion so that extrapolation to 3-D geometry is straight-forward. The behavior of strong shocks born out of the target blast is captured accurately by a second order Godunov algorithm. Diffusive terms (viscosity and thermal conductivity) are included and can depend on state variables (e.g., local temperature). The uniform accuracy throughout the fluid domain is obtained through adaptive mesh refinement. This is essential as the width of the shock region is usually several orders of magnitude smaller than the chamber dimensions. The arbitrary chamber geometry is incorporated into a Cartesian grid and resolved by an embedded boundary method. The details of numerical methods utilized in SPARTAN and the convergence tests are given in Ref. [40]. Example results are shown in Figure 16 for a $154 \mathrm{MJ}$ directdrive target case in a chamber of radius $6.5 \mathrm{~m}$ filled with xenon (with an atom density of $\left.1.6 \times 10^{21} \mathrm{~m}^{-3}\right)$. The simulation results demonstrate the robustness of SPARTAN numerical 
algorithms in studying the highly nonlinear chamber dynamics with fast moving discontinuities.

SPARTAN has been developed to model the chamber dynamics of dry wall concepts. It could be a useful tool if applied to the liquid wall concept also; however, the capability to model liquid-wall specific mechanisms such as ablation, condensation and aerosol formation and behavior, would need to be included.

\section{INSERT FIGURE 16}

\subsubsection{TOPGUN}

A useful model that integrates the coupled transport behavior of gases and aerosols is the TOPGUN code developed at the Idaho National Laboratory [47]. The code was originally developed to simulate plasma-gun experiments used to generate and characterize aerosols representative of those produced in the disruption of a tokamak fusion reactor. It has recently been modified to study the generation and behavior of aerosols in the context of IFE chamber clearing [27]. TOPGUN includes a 1-D gas dynamics model for a multiple charge species, single component gas, and 0-D aerosol dynamics model coupled to the gas dynamics model by source/sink terms in mass, energy, and momentum, in addition to cell-convection terms for aerosol transport. The solution algorithm incorporates semi-implicit differencing and sub-cycling of the aerosol model within the gas dynamics solution. Although the gas dynamics model is not as suitable for IFE post-shot chamber conditions as other codes (e.g. SPARTAN and BUCKY), the essential features are present that provide a reasonable estimate of conditions for aerosol formation and growth. The solution technique of TOPGUN does permit modifications and extension of the aerosol mode, allowing exploration of mechanisms relevant to chamber dynamics and clearing. Studies preformed to date have incorporated aerosol transport mechanisms discussed in Sections 3.1.4 and 3.2.2. Results of an example TOPGUN simulation for aerosol formation are shown in Figure 17 [27]. Aerosol size distributions at various times are shown for the central region of an example 
$6.5 \mathrm{~m}$ spherical chamber protected by a thick wall of liquid lead. The simulation showed that material vaporized from the wall expands, cools, condenses, grows, and is convected through the chamber, giving the size distributions shown in the figure. Benchmarking of TOPGUN was performed using aerosol size data from the plasma gun experiment [47], with TOPGUN reasonably matching the measured size distributions of particles $<\sim 50$ $\mu \mathrm{m}$. Components of the TOPGUN aerosol model would be very useful in a more comprehensive IFE chamber dynamics simulation code, and implementation of similar models into the next version of TSUNAMI is being considered.

\section{INSERT FIGURE 17}

Key R\&D needs to help in better modeling and understanding aerosol formation and behavior in a thick liquid wall chamber should include:

(1) Extending models to include conditions more relevant to thick liquid wall chamber conditions, such as ionization and cooling plasma effects for gas dynamic and aerosol nucleation, multiple component materials, and impact deposition and reflection of aerosols.

(2) Performing experiments to verify simulations of aerosol dynamics, and study relevancy of other proposed condensation mechanisms, such as laser ablation in background plasma for ion-induced nucleation studies (with extension to multiple components), high velocity aerosol impact on liquid surfaces, and condensation behavior of pure flibe.

\subsection{Experiments}

Experimental facilities with capabilities to simulate TLW chamber dynamics include: laser/material interaction laboratory (e.g. UCSD); X-ray facility (e.g. XAPPER at LLNL); Z facility at the Sandia National Laboratories (SNL); plasma gun facility (e.g. UCLA); shock tube facility (UW); and hydraulic facilities at UCB and the Georgia Institute of Technology (Georgia Tech.). Again, this list is not exclusive as there may be other facilities that could be adapted to help address some of the key issues associated 
with liquid wall chamber dynamics. However, they provide a good snapshot of current experimental capabilities that could be utilized to help understand and solve those issues.

\subsubsection{Laser Simulation Experiments (e.g. at UCSD)}

Short-pulse lasers can provide heat fluxes with prototypical energy density and time scale for simulating the thermal, mechanical and phase-change response of liquid wall IFE chambers. Their advantages include ease of operation, low cost, and flexibility. Typically, a laser will be directed at a planar sample which represents only a portion of the wall of a chamber. Some of the light will be absorbed very close to the surface (the remainder is reflected); typical absorption depths for metals are of the order of several nanometers. The intensities needed to reach IFE-relevant temperatures are quite modest, such that laser-induced breakdown is usually avoidable.

The small absorption depth of a laser is one of the key concerns with the fidelity of the simulation and, as such, laser simulation would be better suited to IFE cases with shorter ablation depth (e.g. for larger chambers, $\mathrm{R} \sim 5 \mathrm{~m}$ or larger). Figure 18 shows a thermal analysis of a $\mathrm{Pb}$ wall following a laser pulse as compared with a burst of X-rays. The laser energy has been scaled in order to match the late-stage thermal response of the Xray case. The $\mathrm{X}$-ray spectrum was obtained from a $458 \mathrm{MJ}$ indirect-drive target spectrum [48]. The figure shows that during the pulse the surface temperature rises about a factor of two higher in the case of the laser irradiation, but only in a very thin region $(\sim 100 \mathrm{~nm})$. After the pulse terminates at $\sim 2$ ns, the near-surface temperature equilibrates very quickly, and the resulting thermal diffusion wave is nearly identical in the two cases.

\section{INSERT FIGURE 18}

In an evaporating system, it is perhaps more important to maintain similarity in the mass which is evaporated or explosively ejected. Since the laser is absorbed closer to the surface, less mass will evaporate for a given fluence $\left(\mathrm{J} / \mathrm{cm}^{2}\right)$, but that mass will absorb more energy. It will come off hotter and be more prone to explosive boiling. A 
qualitative comparison can be made between the conditions predicted to occur following an IFE explosion and the conditions often observed in laser ablation plumes. Laser ablation plumes with initial temperature of the order of 1-20 eV and density of the order of $10^{21} \mathrm{~cm}^{-3}$ are easily obtained [49]. These temperatures and densities are very similar to those expected following an ablation plume resulting from IFE X-rays, as summarized in Table VI. Ablation plumes are highly dynamic; as the plume expands, the temperature rapidly falls below $1 \mathrm{eV}$ (after 50-100 ns), the density falls, and the vapor interpenetrates the surrounding medium.

When using a simulated energy source, the most important criterion is to clearly understand the physics involved so that the results can be properly scaled. One needs to exercise caution when simulating X-rays with a laser, but roughly similar material conditions can be achieved such that meaningful experiments are possible.

\section{INSERT TABLE VI}

\subsubsection{X-Ray Facility (e.g. XAPPER)}

The XAPPER X-ray damage experiment, which is located at Lawrence Livermore National Laboratory, is capable of providing high flux bursts of soft X-rays at repetition rates of up to $10 \mathrm{~Hz}$. Fluences can be as high as $\sim 5 \mathrm{~J} / \mathrm{cm}^{2}$ in a $40 \mathrm{~ns}$ pulse with an average $\mathrm{X}$-ray energy of $\sim 120 \mathrm{eV}$. The high repetition rates offers shots on demand as well as the possibility of obtaining favorable statistics by performing a large number of shots. XAPPER has demonstrated continuous $10 \mathrm{~Hz}$ operation for $2 \times 10^{5}$ pulses and can be operated for $\sim 10^{7}$ pulses prior to requiring minor maintenance. Additional details on the XAPPER experiment can be found in References [50] and [51].

To a somewhat lesser extent than for the laser, the soft X-ray energy is absorbed close to the material surface. For example, the mean free path of $120 \mathrm{eV} \mathrm{x}$-rays is $75 \mathrm{~nm}$ in flibe. This longer deposition length may enable the experiment to more closely follow the timetemperature history expected from an actual IFE exposure. 
XAPPER would be well suited for study of liquid wall ablation and condensation. Implementation of liquid wall experiments would require several, relatively inexpensive modifications. First, a small, custom optic would be designed and fabricated. Such an optic would deliver a larger, flat-topped X-ray pulse as opposed to the small, highly peaked pulse currently available. Second, a small target chamber (15-20 cm diameter) would be designed and built. Instrumentation might include residual gas analysis (already available on XAPPER) and other diagnostics similar to those described below in the plasma gun facility section. Early experiments would begin with frozen materials, but later experiments could take advantage of currently available heaters to provide a liquid target.

One concern in the testing of liquid wall vaporization and condensation is the initial state of the liquid. Some argue that a flowing or continuously renewed surface is required in order to obtain results that are truly relevant to thick-liquid wall systems [52]. It is possible to design a very modest (small) flow loop that could meet this requirement. Clearly, such experiments would be more complicated, and thus, more expensive to conduct.

\subsubsection{Z Facility}

The $\mathrm{Z}$ machine at Sandia National Laboratories is the world's most powerful X-ray machine. A multi-wire fast z-pinch load on $\mathrm{Z}$ routinely produces up to $1.8 \mathrm{MJ}$ of $\mathrm{X}$-rays at a power level of about $230 \mathrm{TW}$, and at fluences that can exceed $3,000 \mathrm{~J} / \mathrm{cm}^{2}$ on a single-shot basis. Testing of materials on $\mathrm{Z}$ is done on an add-on basis, when available on scheduled shots. By varying the sample location, and using apertures and filters, an extremely uniform X-ray fluence can be obtained. At distances of the order of $50 \mathrm{~cm}$ from the z-pinch source, fluences can be produced in the range of $1-50 \mathrm{~J} / \mathrm{cm}^{2}$ over a sample area of several $\mathrm{cm}^{2}$ or more. With appropriate $\mathrm{X}$-ray filters, an X-ray spectrum above $1 \mathrm{keV}$ is routinely attainable at IFE-relevant fluences. Through use of apertures, 
filters, and, possibly, shutters, the effects of debris from the z-pinch can be minimized. Post-shot diagnostics include surface profilometry measurements, SEM, ion milling, etc.

Recently, $\mathrm{Z}$ has been used to examine solid material ablation at relatively high fluences $\left(10^{\prime}\right.$ s of $\left.\mathrm{J} / \mathrm{cm}^{2}\right)$, to examine solid surface roughening with no net ablation at smaller fluences ( $\mathrm{a}$ few $\mathrm{J} / \mathrm{cm}^{2}$ ), and to establish the threshold for solid surface roughening (which is of the order of $1 \mathrm{~J} / \mathrm{cm}^{2}$ or less). In this role, $Z$ has been very useful in testing many candidate first wall solid materials (C, W, W/Re, etc.). For liquid-wall material testing, $\mathrm{Z}$ is uniquely qualified to produce fluences at all of the levels that would occur in a liquid-wall power plant - from fluence levels of $1 \mathrm{~J} / \mathrm{cm}^{2}$ to model the effects of wetted walls at several meters from the target, all the way up to fluence levels of $2000 \mathrm{~J} / \mathrm{cm}^{2}$ to model the closest thick-liquid walls (at about $50 \mathrm{~cm}$ from the target) envisioned for IFE power plants. The capability for producing, testing, and diagnosing liquid targets on $\mathrm{Z}$ could presumably be done when support becomes available.

Currently, the capability for testing heated samples (on add-on shots on $\mathrm{Z}$ ) at temperatures up to $1200^{\circ} \mathrm{C}$ is available. A sample test area diameter of $1 \mathrm{~cm}^{2}$ can be shared by 4 samples on each shot. The X-ray fluence is adjustable by varying the distance from the z-pinch, and the X-ray spectrum can be manipulated through the use of X-ray filters.

\subsubsection{Plasma Gun Facility (e.g. UCLA)}

The objective of the UCLA plasma gun experimental facility is to study vapor condensation and chamber clearing rates in IFE relevant conditions, using prototypical materials. The main attractiveness of the facility is the capability of generating large amount of excited vapors in relatively short periods $\left(10^{-4} \mathrm{~s}\right)$, allowing the decoupling of the generation, injection and expansion of the vapors from the chamber clearing process $\left(10^{-1} \mathrm{~s}\right)[53]$. With the electro-thermal source typically operating at $30 \%$ of its full capability, about 0.4 grams of the material of interest can be ablated. This allows one to produce the same initial vapor density in a 5-liter test chamber as expected in the 
HYLIFE-II chamber from the vaporization of the liquid pocket surfaces [6]. Another fundamental characteristic of the facility is the capability of testing IFE prototypical materials, and in particular flibe. Although the facility has been mainly operated using only the non-toxic component of the molten salt $(\mathrm{LiF})$, preliminary runs have demonstrated the possibility of using flibe in the source [54].

The facility has been mainly designed and scaled to simulate the IFE chamber clearing process. For this purpose, the condensation chamber is equipped with sensors that are capable of measuring the pressure at different locations over the wide range that characterize the clearing process. A steady-state residual gas analyzer is then used to evaluate the presence of non-condensable impurities. Typical results for $\mathrm{LiF}$ vapor and $\mathrm{CH}_{4}$ vapors are presented in Figure 19. Time resolved spectroscopic analysis of light emission from the excited gas coupled with Langmuir probes is currently tested to better characterize the local gas thermodynamic properties (density and temperature). The condensation chamber is also equipped with a photo-diode system to measure the velocity of the shock front as it first enters the chamber. This is done mainly to compare gas velocity measurements with the TSUNAMI code simulations of vapor propagation through the chamber. Another inherent capability of the condensation chamber regards the investigation of in-flight condensation and aerosol formation, which would just require additional diagnostics for the time resolved detection of droplet formation. Currently only passive diagnostics are employed, such as collecting buttons for surface post-analysis.

\section{INSERT FIGURE 19}

\subsubsection{UW Shock Tube Facility}

The UW shock tube (shown in Figures 20 and 21) is vertical, with outer round and inner square cross section $(25.4 \mathrm{~cm}$ by $25.4 \mathrm{~cm}$ ) so as to have parallel walls to perform flow visualization anywhere along the tube. The facility's structural capability allows for the production of Mach-5 shock waves in air at atmospheric pressure. The driver section is 
$2.0 \mathrm{~m}$ long; the driven section is about $7.6 \mathrm{~m}$ long and composed of modular segments of different lengths that can be arranged at will. Special sections are available to contain one or more water layers and one or more metal cylinders and to perform flow visualization. These sections can be mounted anywhere along the length of the tube. Available instrumentation includes piezoelectric pressure transducers, several high speed data acquisition channels, CW and pulsed lasers, several CCD cameras (both scientific grade and high speed), and a large variety of optical components.

\section{INSERT FIGURES 20 AND 21}

The facility is very well suited to study the effect of a shock wave upon one or more stationary liquid layers with either circular or rectangular cross section. Experiments so far have concentrated on the measurements of the $x-t$ trajectories of the shock and of a single, initially stationary, water layer and on the changes in the spatial and temporal distributions of pressure around a solid cylinder with and without a stationary water layer placed about four diameters above the cylinder.

Experiments in the near to mid-term future will concentrate on two main areas: the behavior of multiple, stationary water layers subjected to impulsive acceleration and their ability to reduce the peak pressure load on a single cylinder or bank of cylinders; and the mechanisms that lead to the fragmentation and disruption of the water layer and possible formation of aerosols. Longer-term plans include experimental campaigns to study the interaction of a shock with single or multiple liquid jets with both circular and rectangular cross sections (simulating, for example, liquid impact on HIBALL flow tubes or HYLIFE jets).

\subsubsection{Existing hydraulic facilities}

Existing hydraulic facilities at UCB and at Georgia Tech. can be utilized to help understand the dynamics of the liquid jet formation for a thick liquid wall chamber such as HYLIFE-II that utilizes oscillating jets to form a pocket prior to each shot as well as an 
array of crossing jets in front of beam ports (see Fig. 2). A key issue is linked to the quality of the jets to avoid droplets formation in particular as the nozzle starts to wear out or if small-scale impurities or deposits cause local nozzle obstruction.

\subsubsection{UCB Hydraulic Test Facilities}

Several hydraulic facilities at UCB are used to help understand the dynamics of thickliquid jet formation, disruption, and recovery. These are described below. In addition, UCB's experimental activities include the investigation of the properties of both flibe and flinabe and, in particular, of their vapor pressures as a function of temperature [55]. The attractiveness of flinabe stems from its lower melting point and its low vapor pressure at temperatures below the melting point of flibe, which makes it compatible for use in neutralized ballistic transport beam tubes $[7,21,25]$.

\section{Target Chamber Liquid Jet Structures}

As presented in Sections 2.1 and 3.2.3, the RPD thick-liquid structures consist of an oscillating voided pocket, cylindrical jets, and beam-line vortices. All three kinds of jet structures (see Fig. 22) have been demonstrated experimentally in scaled experiments at UCB. Of particular interest for fusion systems are the vortex flow surface roughness, surface renewal, and droplet ejection rate that remain to be characterized. Particle image velocimetry (PIV) will provide detailed velocity and turbulence information on the vortex flow [55].

\section{INSERT FIGURE 22}

As an alternative to HYLIFE-II-like chambers, which is the main focus of this paper, another thick-liquid chamber, the newly introduced "vortex chamber," is being investigated $[42,55]$. The vortex chamber would consist of a neutronically thick swirling layer that runs over the structural first wall. A thick-liquid IFE chamber, in which beam final-focus and transport in the chamber would be performed by a set of solenoids, is depicted schematically in Fig. 23. 
UCB demonstrated the feasibility of establishing and controlling the thick-liquid layer in a cylindrical chamber, as shown in Fig. 24 . This work is still at an early stage and further effort is required to adapt this work to curved geometries for use in IFE and, potentially, MFE chambers.

\section{INSERT FIGURE 23}

\section{INSERT FIGURE 24}

\section{Liquid Jet Response to Ablation and Pocket Pressurization Impulse Load}

The Vacuum Hydraulic EXperiment (VHEX), shown on the right-hand side of Fig. 22, was designed to study thick-liquid jet structure formation, disruption, and recovery. Past work focused on the disruption and recovery of the thick-liquid pocket [28]. Experiments conducted on VHEX demonstrated the first oscillating pocket and the timeliness of the pocket reformation following the disruption caused by the ablation and pocket pressurization impulse. The validity of the snowplow model of jet disruption was confirmed, as shown in Fig. 25.

\section{INSERT FIGURE 25}

Current work includes upgrading the firing mechanism of VHEX for higher fidelity experimental simulations of scaled pocket disruption and reformation. The VHEX used blank shotguns to simulate the IFE impulse. High explosives are now being used for a better repeatability and quality of the impulse delivered to the jets [55]. New disruption experiments will use a scaled pocket that includes oscillating voided slab jets and a partial array of cylindrical jets. This work aims at proving that the pocket and cylindrical jets can be restored before the next shot and assessing how the jets break up into droplets. Use of a different nozzle and other minor modifications to the facility will allow simulation of a Z-IFE thick-liquid curtain as well. 


\subsubsection{Georgia Tech Hydraulic Test Facilities}

Three IFE-relevant hydraulic test facilities are available at Georgia Tech: two of them, the Forced Film Test Facility; and the Porous Wetted-Wall Test Facility are applicable to a wetted wall concept; the third one, the Large-Scale Hydraulic Test Facility is applicable to a thick liquid wall concept and is described in this section.

The Georgia Tech Large-Scale Hydraulic Test Facility is a re-circulating flow loop for the study of turbulent water jets issuing into ambient air (see Figure 26). Rectangular jets with Reynolds numbers $R e=1.0 \times 10^{4}-1.5 \times 10^{5}$ and Weber numbers $W e=5.0 \times 10^{2}-$ $2.4 \times 10^{4}$ can be examined. The jets can be either stationary or oscillated at prescribed frequencies (up to $10 \mathrm{~Hz}$ ) and amplitudes, with Strouhal number $S t=6.0 \times 10^{-3}-6.0 \times$ $10^{-2}$. An external chiller allows the system to operate isothermally for an indefinite period of time. The end flow elements, flow conditioner (E) and nozzle (G), are removable for replacement or modification. Initial conditions may also be modified using an external boundary layer cutter (I).

\section{INSERT FIGURE 26}

Several experimental setups for the study of different flow phenomena are available at this facility. Initial conditions are evaluated by measuring velocity and turbulence intensity profiles just upstream of the nozzle exit using laser-Doppler velocimetry (LDV). Planar-laser induced fluorescence is used to examine the free surface of the jet at different downstream location. The water in the test loop is dyed with a fluorescing salt and illuminated by a laser sheet $(\mathrm{L})$. A CCD camera (M) images the free surface as the interface between fluorescing water (bright) and non-fluorescing air (dark). Jet crosssection, free-surface fluctuations, and average free-surface position are all quantifiable by this technique. The primary turbulent breakup of the jet is estimated with a mass collection apparatus $(\mathrm{K})$. Cuvettes of known mass are positioned at a given distance away from the free surface for a specified period of time. The mass collected in the cuvettes then gives a measure of mass of droplets ejected at the free surface. 


\section{CONCLUSIONS AND FUTURE R\&D}

TLW chambers offer the advantage of moderating the high-energy neutron output from the target, thereby reducing the radiation damage rate and leading to longer lifetimes for the first wall and blanket structures. By utilizing a lithium-containing liquid, such as the molten salts flibe or flinabe, the thick liquid wall also serves as the tritium-breeding blanket and the primary coolant since it directly absorbs all of the short range target emissions ( $\mathrm{X}$-rays and target debris) and the majority of the neutron energy. The key issues to be addressed to realize the potential of TLW chambers can be summarized in three primary categories: (1) issues related to the repetitive nature of IFE, including liquid wall response to the pulsed energy release and recovery of chamber conditions between pulses (reformation of the protective liquid configuration, clearing of drops and vapor that could interfere with the next shot); (2) issues related to shock mitigation, including the ability of multi-layer thick liquid wall configurations to attenuate shocks and thus protect the structural wall from possible damaging effects of shocks; and (3) issues related to the use of molten salt (the preferred liquid) or liquid metal, including material compatibility (corrosion), target debris transport and removal, tritium recovery, heat transport and power conversion.

This paper has focused on the first category. The chamber dynamics in a thick liquid wall concept such as HYLIFE-II are governed by a number of different mechanisms occurring at different times in the liquid wall and chamber following the target micro-explosion. The photon energy deposition at very short times dictates the ablation mechanisms, including explosive boiling and, possibly, spalling created by the resulting impulse on the wall. Isochoric heating from neutrons might create additional jet break up depending on the target yield and jet location. The behavior of the ablated material in terms of aerosol formation and then transport is an important issue as any aerosol remnant after the pocket reformation or at the axial open ends of the pocket might affect the driver performance. The liquid jet quality is also important as any spray could provide additional aerosol 
material and the behavior of the oscillating jets in forming the pocket prior to each shot must be also highly reliable. Typical capabilities of existing models and experiments in addressing these issues have been described with a view of helping to better recognize where there are gaps in current understanding and capabilities and where future R\&D effort should be directed.

The information from this paper is intended to help in the assessment of R\&D needs for TLW chambers, such as the recent one carried out by IFE researchers as part of a Virtual Laboratory for Technology (VLT) exercise [56]. The list of the R\&D required to address and resolve key issues for TLW chambers included the following items related to the TLW chamber dynamics, which also reflects well the observations from this paper.

1) Fundamental science research on various aspects of thick liquid walls will be needed (e.g., turbulence effects on free surfaces, shock propagation and mitigation, aerosol formation and evolution, etc.). These are typically university scale tests and research, which have proven valuable in advancing thick liquid wall chambers to their current state.

2) Hydraulics Test Facility - to demonstrate the type of flow configurations needed for TLW chambers at $\sim 1 / 4$ scale. A simulant fluid (e.g., mineral oil or water) would be used to minimize costs. The facility would simulate (e.g., by using high explosive detonations) the disruption of the flow by fusion energy pulses to study the ability to clear the chamber of drops in time for the next shot. The facility would also be used to study and validate scaled shock mitigation techniques.

3) Chamber Dynamics Test Facility - to study the dynamics of vaporization and condensation of molten salt or liquid metal in the chamber with a focus on aspects that are unique to working fluid and cannot be simulated in the hydraulic test facility.

Other R\&D items included liquid test loops and heat transfer component facilities. While much more work is needed to define experiments, design the test facilities and estimate construction and operating costs, preliminary estimates from the VLT assessment indicate that this type of $R \& D$ could be conducted at the $\sim \$ 10 \mathrm{M} / \mathrm{yr}$ level. 


\section{ACKNOWLEDGEMENT}

This paper was motivated by the informative presentations and discussion at the 2003 ARIES Town Meeting on Liquid Wall Chamber Dynamics [8]. The authors are grateful to all participants of the Town Meeting and to the ARIES-IFE Team for their inspiring contributions to the discussion. This paper was supported in major part by grants from the U.S. Department of Energy, in particular under Contract DE-FC03-95ER54299. 


\section{References}

1. A.P. FRASS, “The BLASCON - An Exploding Pellet Fusion Reactor," ORNL report TM-3231, (1971).

2. R.J. BURKE, "Outline for a Large-Pulse Electron Beam Ignited Fusion Reactor," ANL report CTR/M-31 (1974).

3. W.R. MEIER and J.A. MANISCALCO, "Reactor Concept for Laser Fusion," UCRL-79694, LLNL (1977).

4. M.J. MONSLER et al., "Electric Power from Laser Fusion: the HYLIFE Concept," LLNL report UCRL-812259, Rev. 1 (1978).

5. M.J. MONSLER and W.R. MEIER, "A Conceptual Design Strategy for LiquidMetal-Wall Inertial Fusion Reactors," Nuclear Eng. and Design 63, 289 (1981).

6. R. MOIR, et al., "HYLIFE-II: a Molten-Salt Inertial Fusion Energy Power Plant Design - Final Report," Fusion Technology, 25, 5 (1994). See also R. W. MOIR, "Improvements to the HYLIFE-II Inertial Fusion Power Plant Design," Fusion Technol. 26, 1169 (1994).

7. S. YU, W.R. MEIER, et al., "An Updated Point Design for Heavy Ion Fusion," Fusion Science and Technology, 44, 266 (2003).

8. available at: http://aries.ucsd.edu/ARIES/MEETINGS/

9. F. NAJMABADI, A. R. RAFFRAY, et al., "Operational Windows for Dry-Wall and Wetted-Wall IFE Chambers," Fusion Science \& Technology, 46 (3), 401 (2004).

10. D. A. CALLAHAN-MILLER and M. TABAK, "Increasing the Coupling Efficiency in a Heavy Ion, Inertial Confinement Fusion Target," Nuclear Fusion, 39 (7), 883, (July 1999).

11. available at http://aries.ucsd.edu/ARIES/WDOCS/ARIES-IFE/SPECTRA/

12. M. ZAGHLOUL and A. R. RAFFRAY, "IFE Liquid Wall Response to the Prompt X-Ray Energy Deposition: Investigation of Physical Processes and Assessment of Ablated Material," accepted for publication in Fusion Science \& Technology (2004). 
13. A.R. RAFFRAY, et al., "Thermo-Fluid Dynamics and Chamber Aerosol Behavior for Thin Liquid Wall under IFE Cyclic Operation," Fusion Science \& Technology, 46 (3), 438 (2004).

14. W. FUCKE and U. SEYDEL, "Improved Experimental Determination of CriticalPoint Data for Tungsten," High Temp.-High Pres., 12, 419, (1980).

15. OSIRIS and SOMBRERO Inertial Confinement Fusion Power Plant DesignsFinal Report. WJSA-9201, DOE/ER/54100-1(March 1992). Also see W.R. MEIER, "Osiris And Sombrero Inertial Fusion Power Plant Designs - Summary, Conclusions, and Recommendations," Fusion Eng. and Design, 25, 145-157 (1994).

16. C. JANTZEN, P. F. PETERSON, "Scaled impulse loading for liquid hydraulic response in IFE thick-liquid chamber experiments," Nuclear Instruments and Methods in Physics Research A, 464, $404-409$ (2001)

17. L. EL-GUEBALY, "Views on Neutronics and Activation Issues Facing IFE Thick Liquid Wall Concept," to be published in Fusion Science \& Technology.

18. B. BADGER, F. ARENDT, K. BECKER et al., "HIBALL - A Conceptual Heavy Ion Beam Driven Fusion Reactor Study," University of Wisconsin Fusion Technology Institute Report, UWFDM-450 (September 1981).

19. M. SAWAN, G. MOSES, and G. KULCINSKI, "Time Dependent Neutronics Analysis for the HIBALL Heavy Ion Beam Fusion Reactor," Nuclear Technology/ Fusion, 2, 215 (1982).

20. J. PERLADO, D. LODI, J. MARIAN, et al., "Time-Dependent Neutronics in Structural Materials of Inertial Fusion Reactors and Simulation of Effect Accumulation in Pulsed Fe and SiC," Fusion Science \& Technology, 43, 384 (2003).

21. C.S. DEBONNEL, et al., "Control of the Heavy-Ion Beam Line Gas Pressure and Density in the HYLIFE Thick-Liquid Chamber," Fusion Engineering and Design, 63-64C, 647 (2002).

22. C.S. DEBONNEL, et al., "X-ray Ablation and Debris Venting for the Heavy-Ion Point Design," Fusion Science and Technology, 44 (2), 274 (2003). 
23. J.C. LIU, Experimental and Numerical Investigation of Shock Wave Propagation Through Complex Geometry, Gas Continuous, Two-Phase Media, Ph.D. thesis, University of California at Berkeley, 1993.

24. J.C. LIU, P.F. PETERSON, and V.E. SCHROCK, "Blast venting through blanket material in the HYLIFE ICF reactor," Fusion Technology, 21, 1514-1519 (May 1992).

25. C.S. DEBONNEL, et al., "Gas Transport and Density Control in the HYLIFE Heavy-Ion Beam Lines," Fusion Science and Technology, 43 (3), 408 (2003).

26. C.S. DEBONNEL, T.X. WANG, M. SUZUKI, E. GARCIA, and P.F. PETERSON, "Visual Tsunami: A Versatile, User-Friendly, Multidimensional Ablation and Gas-Dynamics Design Code," accepted for publication in Fusion Science and Technology (2004).

27. J.P. SHARPE, B.J. MERRILL, D.A. PETTI, "Aerosol Production in IFE Chamber Systems," Fusion Sci. and Tech., 44, 312 (2003).

28. S.J. PEMBERTON, R.P. ABBOTT, and P.F. PETERSON, "Thick-Liquid Blanket Configuration and Response for the HIF Point Design," Fusion Science and Technology, 44 (2), 294-299 (2003).

29. S.J. PEMBERTON, "Thick-Liquid Protection in Inertial Fusion Power Plants," Ph.D. thesis, University of California at Berkeley (2002).

30. S.P. LIN, and R.D. REITZ, "Drop and Spray Formation from a Liquid Jet," Annual Rev. Fluid Mech., 30, 85-105 (1998).

31. K.A. SALLAM, Z. DAI, and G.M. FAETH, "Liquid Breakup at the Surface of Turbulent Round Liquid Jets in Still Gases," Int. J. of Multiphase Flow, 28, 427 449 (2002).

32. S.G. DURBIN, M. YODA, S.I. ABDEL-KHALIK, D.L. SADOWSKI, and T.P. KOEHLER, "The Hydrodynamic Source Term in Thick Liquid Protection," to be published (2004).

33. R.R. PETERSON, et al., "The BUCKY and ZEUS-2D Computer Codes for Simulating High Energy Density ICF Plasmas)," Fusion Tech., 30, 783 (1996).

34. T. MELHORN, "A Finite Material Temperature Model for Ion Energy Deposition in Ion-Driven Inertial Fusion Targets," J. Appl. Phys. 52, 6522 (1981) 
35. J. MACFARLANE and P. WANG, "Radiative Properties and Line Trapping Effects in Post-Explosion Inertial Fusion Plasmas," Phys. Fluids B 3, 3494 (1991).

36. D.A. LABUNTSOV and A.P. KRYUKOV, "Analysis of Intensive Evaporation and Condensation," Int. J. Heat Mass Transfer, 22, 989 (1979).

37. A. T. ANDERSON, "X-Ray Ablation Measurements and Modeling for ICF Applications," Ph.D. Thesis, University of California at Berkeley; also Lawrence Livermore National Laboratory, UCRL-LR-125352 (1996).

38. X.M. CHEN, "A Study of Thermal Hydraulic and Kinetic Phenomena in HYLIFE-II---An Inertial Confinement Fusion Reactor," Ph.D. thesis, University of California at Berkeley (1992).

39. X.M. CHEN, et al., "TSUNAMI Analysis of National-Ignition-Facility 2-D Gas Dynamics Phenomenon," Fusion Technology, 26 (3), 814 (1994)

40. J.M. SCOTT, "X-ray Ablated Plumes in Inertial Confinement Fusion Reactors," Ph.D. thesis, University of California at Berkeley (1998).

41. C.A. JANTZEN, "Gas Dynamics and Radiative Heat Transfer in IFE Chambers with Emphasis on the HYLIFE-I Design," Ph.D. thesis, University of California at Berkeley (2000).

42. C.S. DEBONNEL, S.S. YU, and P.F. PETERSON, "Progress Towards Thick Liquid Fusion Chambers for Assisted-Pinch and Solenoid Focusings," Nuclear Instruments and Methods in Physics Research A, in press.

43. C.S. DEBONNEL, Ph.D. thesis, University of California at Berkeley, in preparation (2004).

44. P. CALDERONI, "On the Study of Vapor Condensation for the Assessment of Inertial Fusion Energy Liquid Chamber Clearing," Ph.D. thesis, University of California at Berkeley (2004).

45. R.W. SCHRAGE, "A Theoretical Study of Interphase Mass Transfer," Columbia University Press, New York (1953).

46. Z. DRAGOJLOVIC, F. NAJMABADI, and M. DAY, "An Embedded Boundary Method for Viscous, Conducting Compressible Flow," submitted to J. Comp. Phys. (2004). 
47. J. P. SHARPE, B. D. MERRILL, and D. A. PETTI, "Modeling of Particulate Production in the SIRENS Plasma Disruption Simulator," Journal of Nuclear Materials, 290-293, 1128 (2001).

48. M.S. TILLACK, et al., "ARIES Inertial Fusion Chamber Assessment," Fusion Technology, 39, 343 (2001).

49. S.S. HARILAL, C.V. BINDHU, M.S. TILLACK, F. NAJMABADI and A.C. GAERIS, "Internal Structure and Expansion Dynamics of Laser Ablation Plumes into Ambient Gases," Journal of Applied Physics 93 (5), 2380 (March 2003).

50. M. McGEOCH, "Radio-Frequency-Preionized Xenon Z-Pinch Source for Extreme Ultraviolet Lithography," Appl. Optics 37, 1651 (1998).

51. J. F. LATKOWSKI, R. P. ABBOTT, S. A. PAYNE, S. REYES, R. C. SCHMITT, and J. A. SPETH, "Rep-Rated X-Ray Damage and Ablation Experiments for IFE and ICF Applications," Inertial Fusion Sciences and Applications, Monterey, CA (2003).

52. R. W. MOIR, personal communication (2002).

53. P.F. PETERSON, "Design Methods for Thick-Liquid Protection of Inertial Fusion Chambers", Fusion Technology, 39 (2), 702 (2001).

54. P. CALDERONI, A. YING, T. SKETCHLEY, and M. ABDOU, "Description of a Facility for Vapor Clearing Rates Studies of IFE Reactors Liquid Chambers," Fusion Technology', 39 (2), 711 (2001).

55. P.M. BARDET, C.S. DEBONNEL, J. FREEMAN, G. FUKUDA, B. SUPIOT, and P.F. PETERSON, "Dynamics of Liquid-Protected Fusion Chambers," Fusion Science and Technology, in press.

56. C. BAKER, "Candidate Technology Test Facilities - MFE and IFE Related," draft report, Virtual Laboratory of Technology, University of California, San Diego (November 11, 2002). 


\section{List of figures}

Figure1 CAD model of HYLIFE-II chamber for the RPD.

Figure 2 Schematic of liquid jets that make up TLW protection.

Figure 3 Photon spectrum from LLNL $458 \mathrm{MJ}$ heavy ion beam indirect-drive target $[10,11]$.

Figure 4 Physical processes in X-ray ablation [12].

Figure 5 Volumetric heat deposition in a flibe wall (or curtain) at $0.5 \mathrm{~m}$ from the microexplosion for the $458 \mathrm{MJ}$ indirect-drive photon spectra, illustrating the region where explosive boiling is likely to occur.

Figure 6 Scaled pressure pulse profile from OSIRIS [15] for flibe liquid wall at radii of $0.5 \mathrm{~m}$ and $3.5 \mathrm{~m}$ for the $458 \mathrm{MJ}$ heavy-ion indirect-drive target. For the $0.5 \mathrm{~m}$ case, the peak pressure $(280 \mathrm{MPa})$ has been scaled down to $1 / 5$ of its value so that it can be represented within the scale of the graph.

Figure 7 Illustration of spalling in a thick liquid wall at a radius of $0.5 \mathrm{~m}$ from the micro-explosion under the scaled initial pressure pulse shown in Fig. 6 (the shock is assumed to move at the speed of sound, C). The ordinate scale on the right-hand-side figures have been magnified for clarity. (Note that these results are only dependent on the distance from the back of the jet and not on the actual thickness of the jet).

Figure 8 Instantaneous nuclear energy deposition at the surface and middle of the $2 \mathrm{~m}$ thick $\mathrm{Pb}-17 \mathrm{Li}$ wall of the HIBALL design.

Figure 9 RPD beamline schematic $[22,25]$. 
Figure 10 (a) Homogeneous nucleation rate (solid lines) and critical particle radii (dashed-lines) for nucleation of $\mathrm{Pb}$ aerosol particles. Note the highly nonlinear behavior at moderate saturation ratios. (b) Mlustration of the time required to nucleate $10^{15}$ particles $/ \mathrm{m}^{3}$.

Figure 11 Time periods for condensation growth of a $1 \mu \mathrm{m} \mathrm{Pb}$ particle as a function of saturation ratio is less than $1 \mathrm{~ms}$.

Figure 12 Coagulation changes the size distribution a number of $1 \mu \mathrm{m}$ aerosol particles in a time frame relevant to intermediate chamber clearing times.

Figure 13 Schematic of voided slab jet compression. Note that the actual slab jets are each made of one sheet jet and an array of cylindrical jets [29].

Figure 14 Vaporized flibe thickness for a thin film as a function of stand-off distance from target.

Figure 15 TSUNAMI density contour plots at various times (the density of the liquid and solid structures is arbitrary low).

Figure 16 Example simulation of an IFE dry wall chamber with Xe as background gas.

(a) Geometry of the chamber with the initial conditions imposed from a 1-D solution obtained by the BUCKY code. The temperature field, as shown in (b) through (d), is given $100 \mathrm{~ms}$ after the target implosion. Solution (b) is obtained by setting diffusive terms to zero. A temperature dependent viscosity is estimated by an empirical law in (c), while the conductivity is neglected. Case (d) features a similar empirical law for conductivity while the viscosity is set to zero. In all the cases the protective gas is Xenon. 
Figure 17 TOPGUN simulation results for aerosol production in a $6.5 \mathrm{~m}$ spherical chamber with a liquid lead wall indicate a significant population of moderately sized aerosol particles existing in the central region of the IFE chamber.

Figure 18 Thermal response of the surface of $\mathrm{Pb}$ exposed to a 2-ns laser pulse and a 2ns soft $x$-ray pulse. The time following initiation of the energy source is 2 $\mathrm{ns}, 5 \mathrm{~ns}, 10 \mathrm{~ns}$ and $50 \mathrm{~ns}$.

Figure 19 Pressure history of Teflon and LiF shots.

Figure 20 UW shock tube schematic.

Figure 21 Image of UW shock tube facility's bottom third.

Figure 22 Three of UCB jet experiments: high Reynolds number cylindrical jets, beam-line vortex flow, and oscillating voided jets [28].

Figure 23 Schematic of the vortex chamber [42]. The first wall is not depicted.

Figure 24 UCB large-vortex experimental setup [55].

Figure 25 Comparison of snowplow compression model with experimental data (the position of the target-facing surface of the slab that makes up part of the oscillating voided jet is shown as a function of time) [29].

Figure 26 The Georgia Tech Large-Scale Hydraulic Test Facility. 
Table I Energy partitioning for $458 \mathrm{MJ}$ heavy ion indirect-drive target $[10,11]$

\begin{tabular}{|l|c|}
\hline & $\begin{array}{c}\text { Heavy Ion Indirect-Drive } \\
\text { Target (MJ) }\end{array}$ \\
\hline X-rays & $115(25 \%)$ \\
\hline Neutrons & $316(69 \%)$ \\
\hline Gammas & $0.36(0.1 \%)$ \\
\hline Burn Product Fast Ions & $8.43(2 \%)$ \\
\hline Total & \\
\hline
\end{tabular}


Table II Theoretical spall strength of flibe [12]

\begin{tabular}{|c|c|}
\hline$T(K)$ & Spall Strength (GPa) \\
\hline 750 & -2.4914 \\
\hline 1450 & -1.4212 \\
\hline 2250 & -0.6848 \\
\hline 2999 & -0.2814 \\
\hline 3749 & -0.0657 \\
\hline
\end{tabular}


Table III Comparison of parameters from OSIRIS [15] and from the assumed 458 MJ heavy ion indirect-drive target

\begin{tabular}{|l|c|c|}
\hline Parameters & OSIRIS & $\begin{array}{c}\text { Present } \\
\text { Study }\end{array}$ \\
\hline Liquid/Structure & Flibe/C & Flibe \\
\hline X-ray yield (MJ) & $129 *$ & 115 \\
\hline Closest distance from target (m) & 3.5 & 0.5 \\
\hline Vaporized mass $\left(\mathrm{kg} / \mathrm{m}^{2}\right)$ & 0.0278 & 0.251 \\
\hline Reactive impulse** $(\mathrm{Pa}-\mathrm{s})$ & 59.0 & 525.6 \\
\hline
\end{tabular}

* X-ray and debris

*** Ablated material velocity $\sim$ sonic velocity $\sim 2094 \mathrm{~m} / \mathrm{s}$ for flibe at $\mathrm{T}_{\text {crit }} \sim 4500 \mathrm{~K}$ [12] 
Table IV. Summary of Simulation Capabilities of Different Models and of Simulation and Measurement Capabilities of Different Experimental Facilities in Addressing IFE Liquid Wall Mechanisms

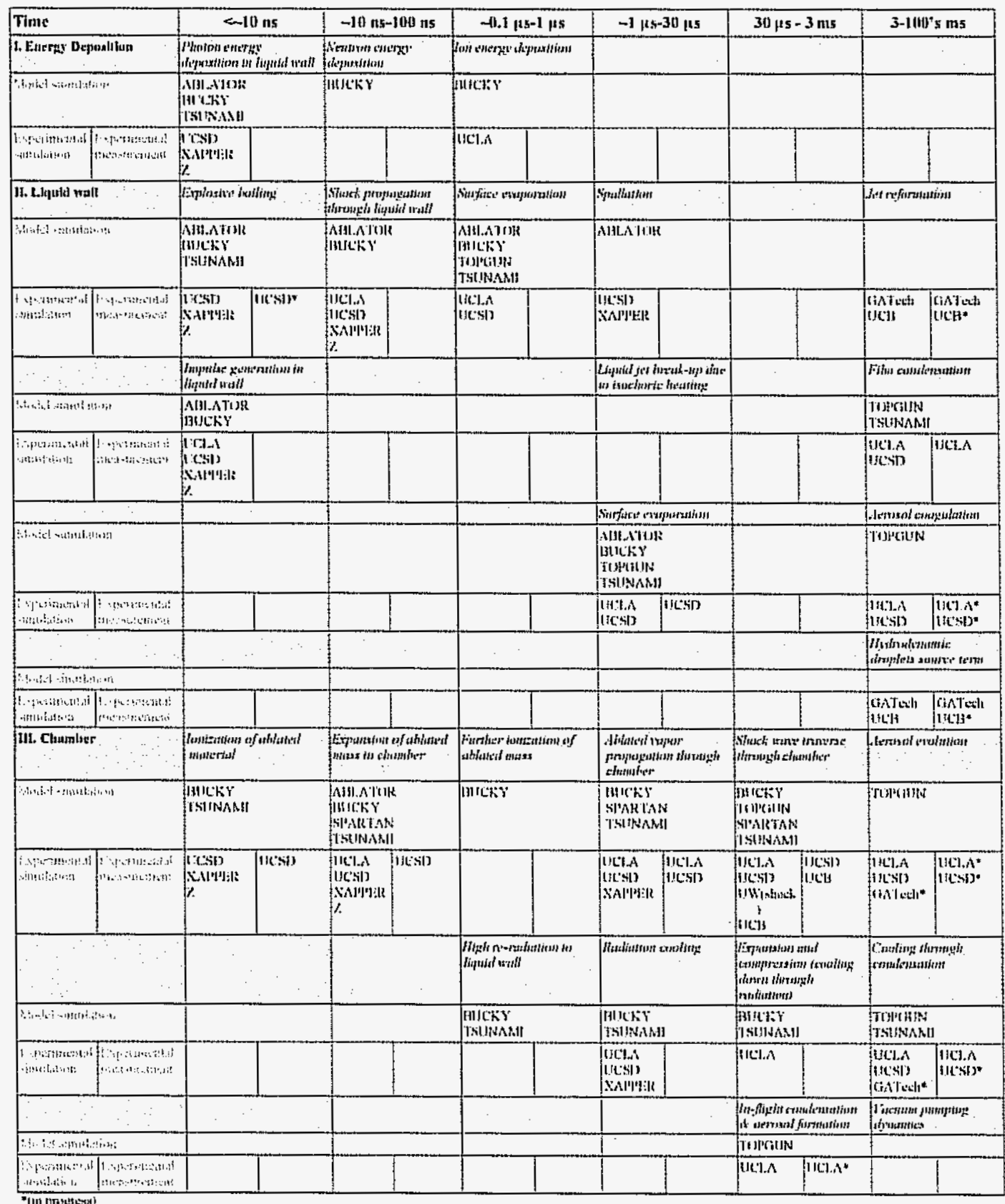


Table V. Comparison of ABLATOR and TSUNAMI results on vaporized flibe thickness during a $40 \mathrm{~ns}$ pulse of a single $113 \mathrm{eV}$ line.

\begin{tabular}{|c|c|c|}
\hline \multirow[b]{2}{*}{$\begin{array}{c}\text { Fluence } \\
\left(\mathrm{J} / \mathrm{cm}^{2}\right)\end{array}$} & \multicolumn{2}{|c|}{ Thickness vaporized } \\
\hline & $\begin{array}{l}\text { Tsunami } \\
\text { (microns) }\end{array}$ & $\begin{array}{c}\text { Ablator } \\
\text { (microns) }\end{array}$ \\
\hline 1 & 0.19 & 0.15 \\
\hline 2 & 0.24 & 0.20 \\
\hline 5 & 0.30 & 0.27 \\
\hline 10 & 0.35 & 0.32 \\
\hline 20 & 0.40 & 0.37 \\
\hline 30 & 0.43 & 0.40 \\
\hline
\end{tabular}


Table VI Comparison of laser induced ablation plume parameters to IFE liquid wall parameters under X-ray energy deposition spectrum from $458 \mathrm{MJ}$ indirect-drive target.

\begin{tabular}{|c|c|c|}
\hline Parameter & $\begin{array}{l}\text { X-rays from HI target } \\
\text { explosion }\end{array}$ & $\begin{array}{l}\text { Laser simulation } \\
\left(\mathbf{1 0}^{7}-10^{10} \mathrm{~W} / \mathrm{cm}^{2}\right)\end{array}$ \\
\hline pulse length & $\sim 2 \mathrm{~ns}$ & $8 \mathrm{~ns}$ \\
\hline attenuation length & $1-5 \mathrm{~mm}$ (Pb/flibe) & $10 \mathrm{~nm}$ \\
\hline ablation depth & $\begin{array}{l}\sim 1-10 \mu \mathrm{m} \text { at } \mathrm{R} \sim 5 \mathrm{~m} \text { (wetted } \\
\text { wall concept) } \\
\sim 100 \mu \mathrm{m} \text { at } \mathrm{R} \sim 0.5 \mathrm{~m} \text { (TLW } \\
\text { concept) }\end{array}$ & $1-2 \mu \mathrm{m}$ (thermal) \\
\hline initial plume: & $\begin{array}{l}\quad<30 \mathrm{eV}^{*} \\
<\mathrm{n}_{\mathrm{c}} \sim 10^{21} / \mathrm{cm}^{3} \\
\text { Not available }\end{array}$ & $\begin{array}{c}1-20 \mathrm{eV} \\
\sim 10^{21} / \mathrm{cm}^{3} \\
0-3\end{array}$ \\
\hline $\begin{array}{c}\text { plume @1 ms: temp. } \\
\text { plasma density } \\
\mathrm{Z}_{\text {eff }} \\
\end{array}$ & & $\begin{array}{c}0.5-1.5 \mathrm{eV} \\
3 \times 10^{18} \mathrm{~cm}^{-3} \\
0-1\end{array}$ \\
\hline background gas density & 0-50 mTorr (@ST) & 0-1 atm (@ST) \\
\hline background gas temperature & $\begin{array}{l}>1000^{\circ} \mathrm{C} \text { (wetted wall } \\
\text { concept) } \\
\sim 500^{\circ} \mathrm{C} \text { (TLW concept) }\end{array}$ & room temperature \\
\hline spot size & $1000 \mathrm{~m}^{2}$ & $1 \mathrm{~mm}^{2}$ \\
\hline geometry & quasi-1D & quasi-1D \\
\hline
\end{tabular}




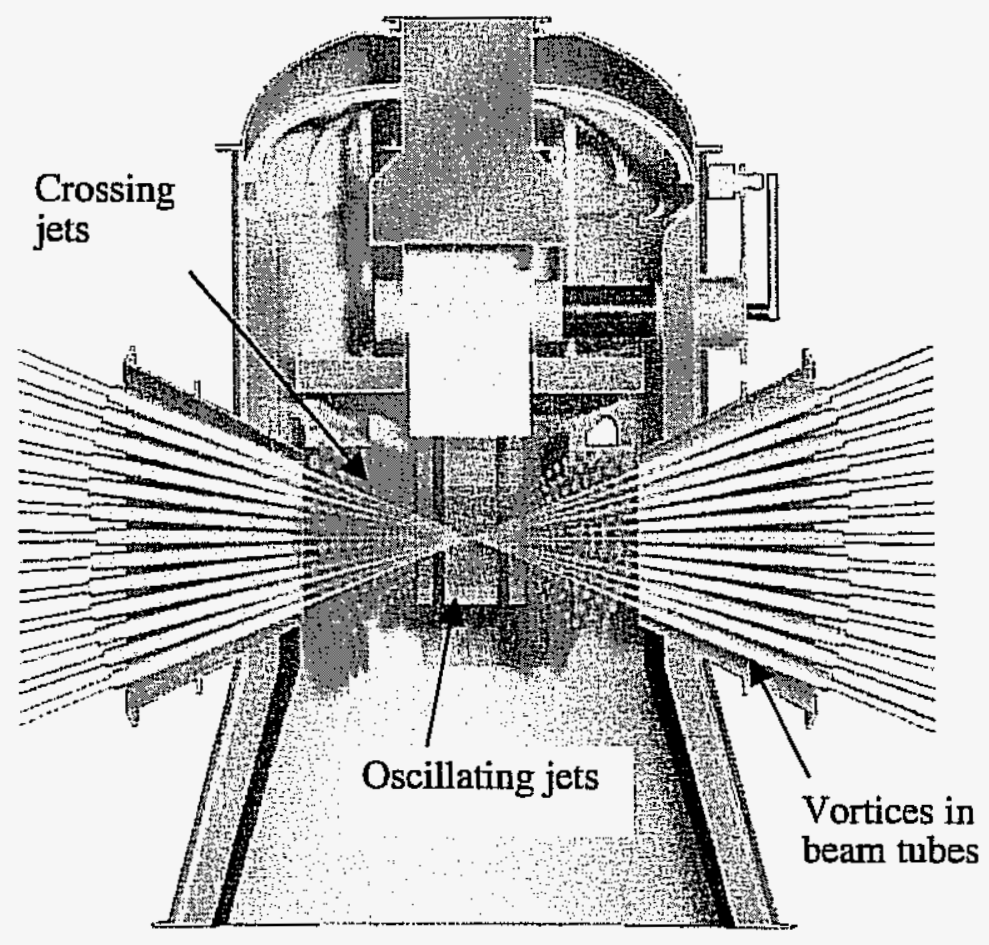

Fig. 1 CAD model of HYLIFE-II chamber for the RPD. 


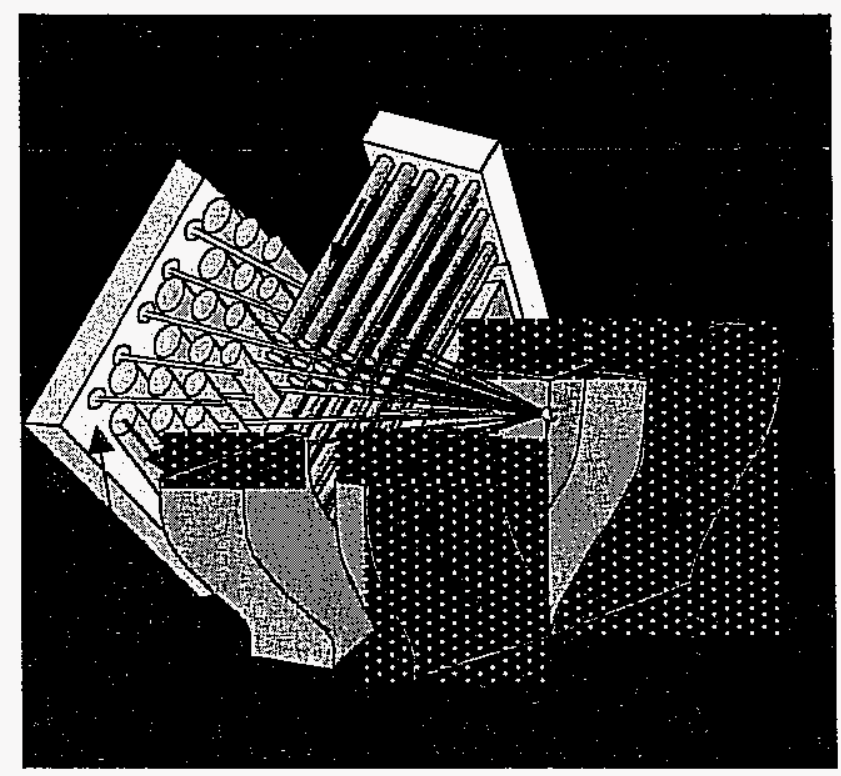

Fig. 2 Schematic of liquid jets that make up TLW protection 


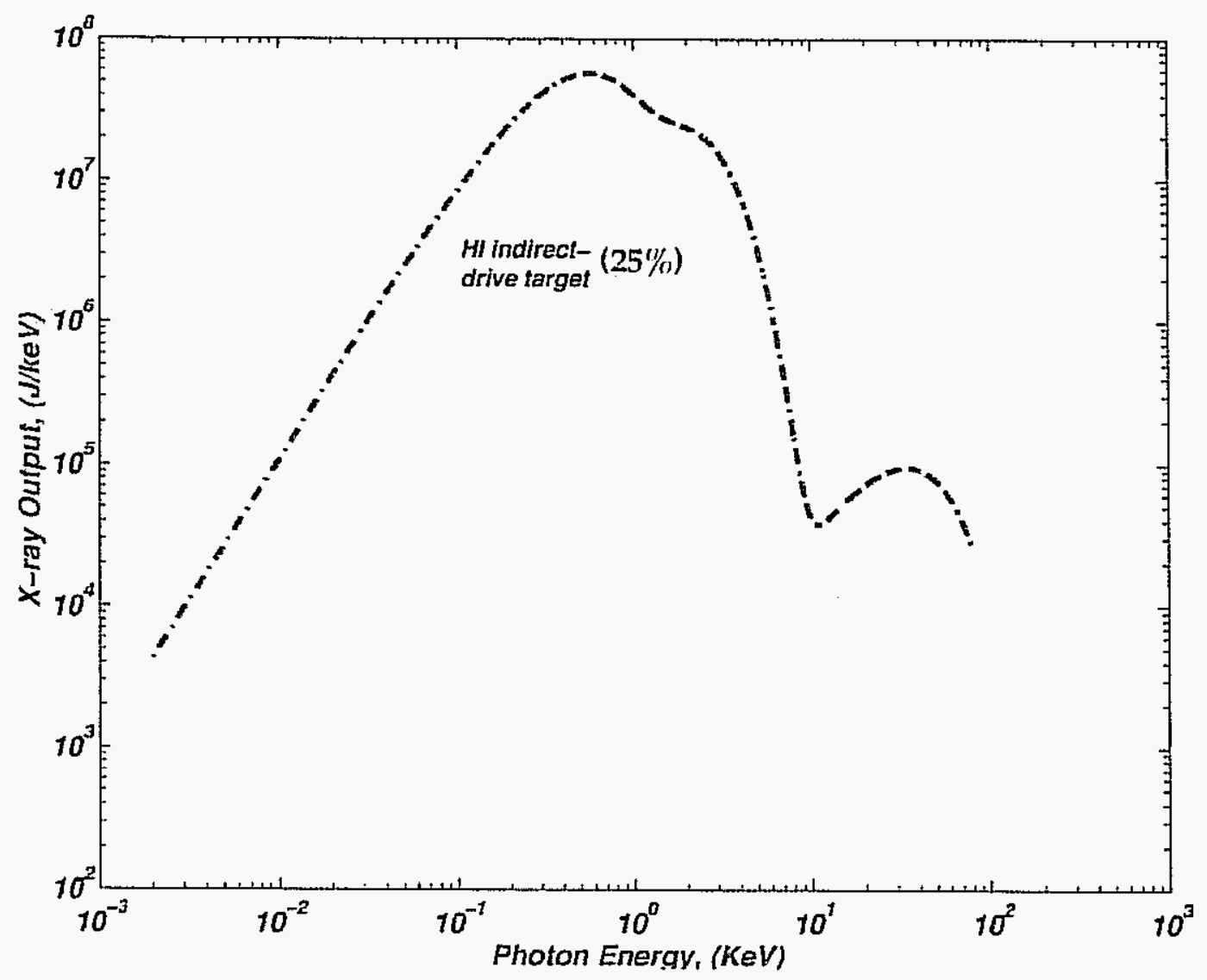

Fig. 3 Photon spectrum from LLNL 458 MJ heavy ion beam indirect-drive target $[10,11]$. 


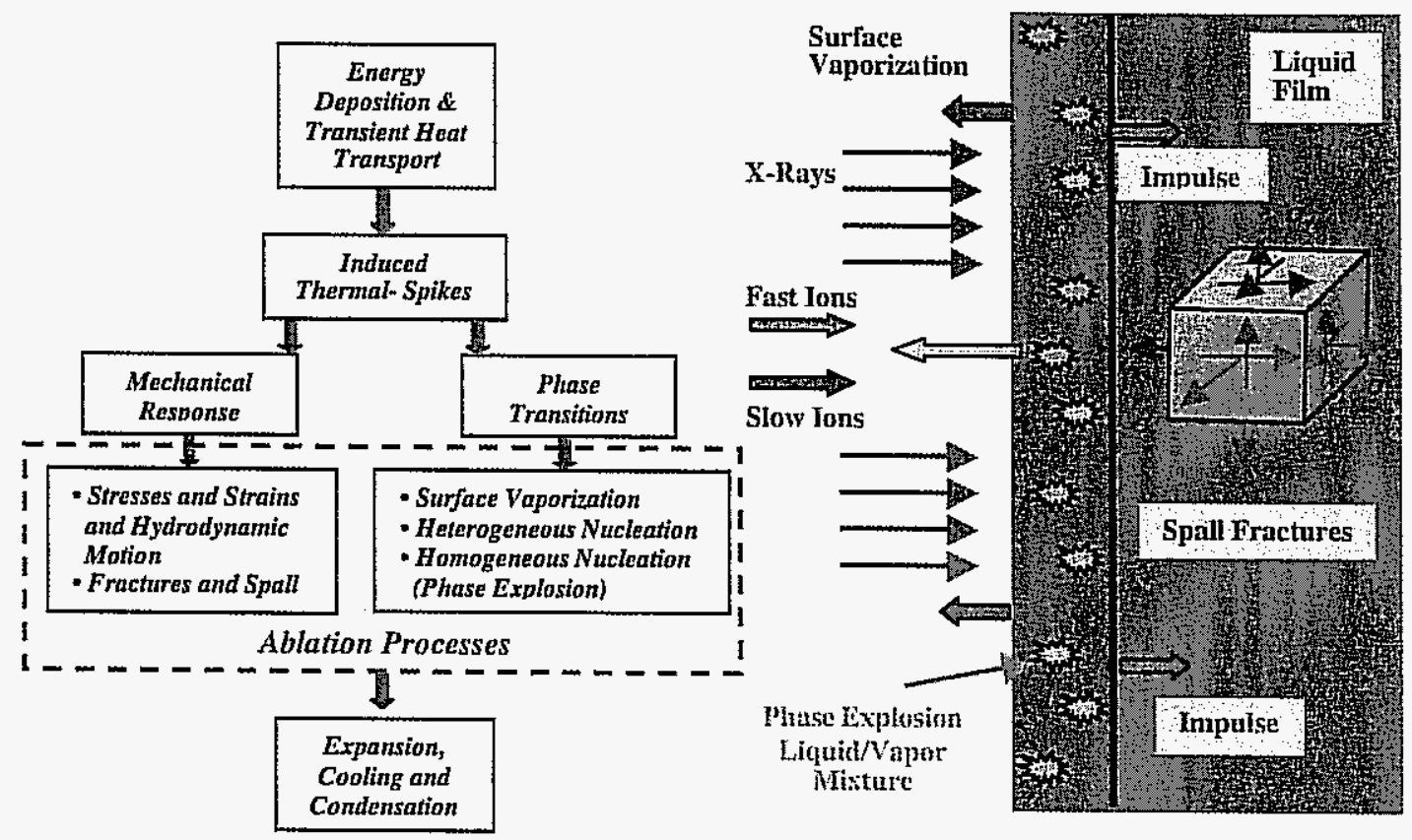

Fig. 4 Physical processes in X-ray ablation [12] 


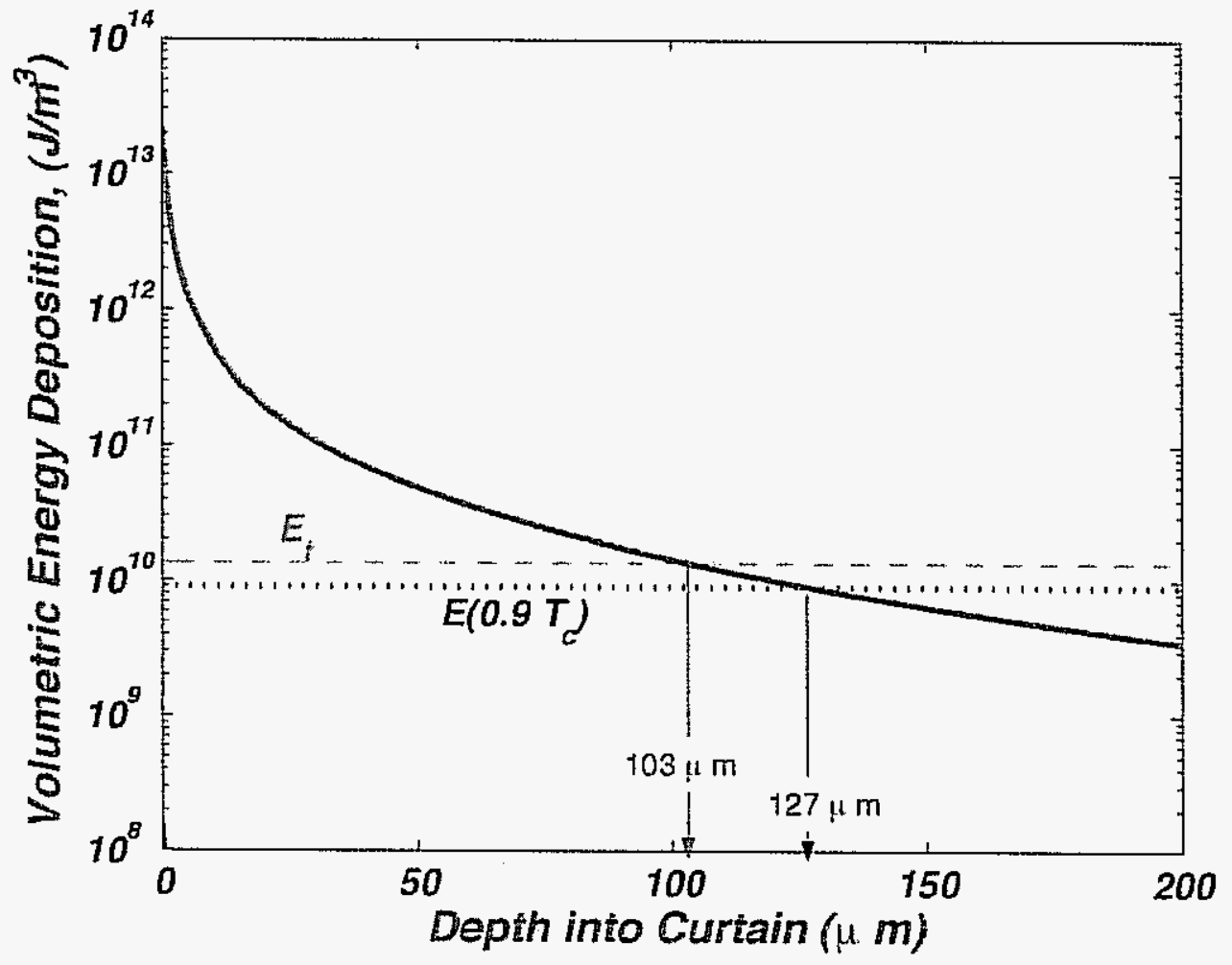

Fig. 5 Volumetric heat deposition in a flibe wall (or curtain) at $0.5 \mathrm{~m}$ from the microexplosion for the $458 \mathrm{MJ}$ indirect-drive photon spectra, illustrating the region where explosive boiling is likely to occur. 


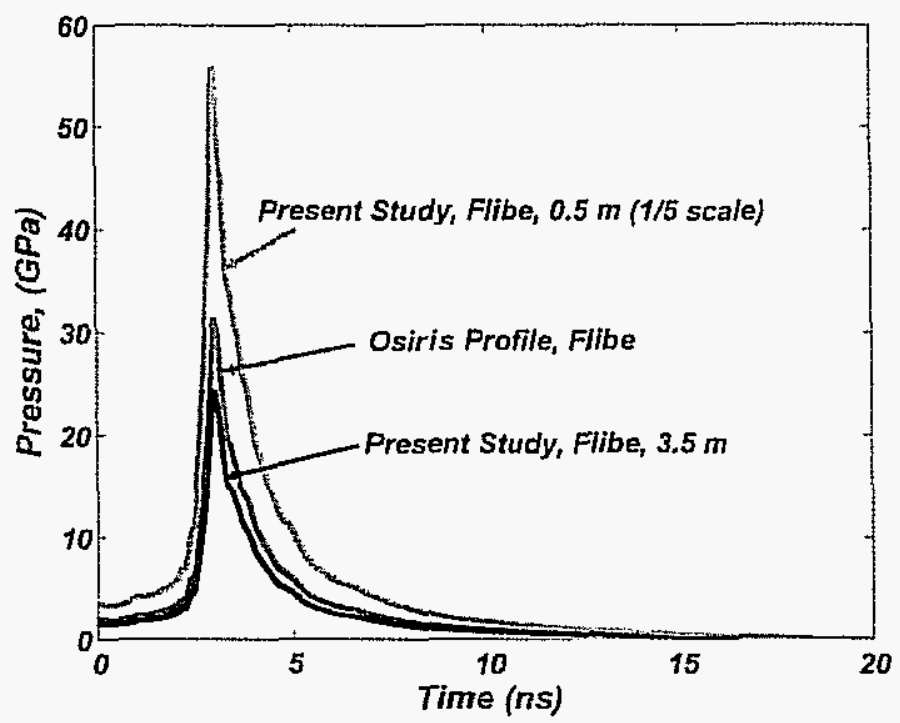

Figure 6 Scaled pressure pulse profile from OSIRIS [15] for flibe liquid wall at radii of $0.5 \mathrm{~m}$ and $3.5 \mathrm{~m}$ for the $458 \mathrm{MJ}$ heavy-ion indirect-drive target. For the $0.5 \mathrm{~m}$ case, the peak pressure ( $280 \mathrm{MPa})$ has been scaled down to $1 / 5$ of its value so that it can be represented within the scale of the graph. 

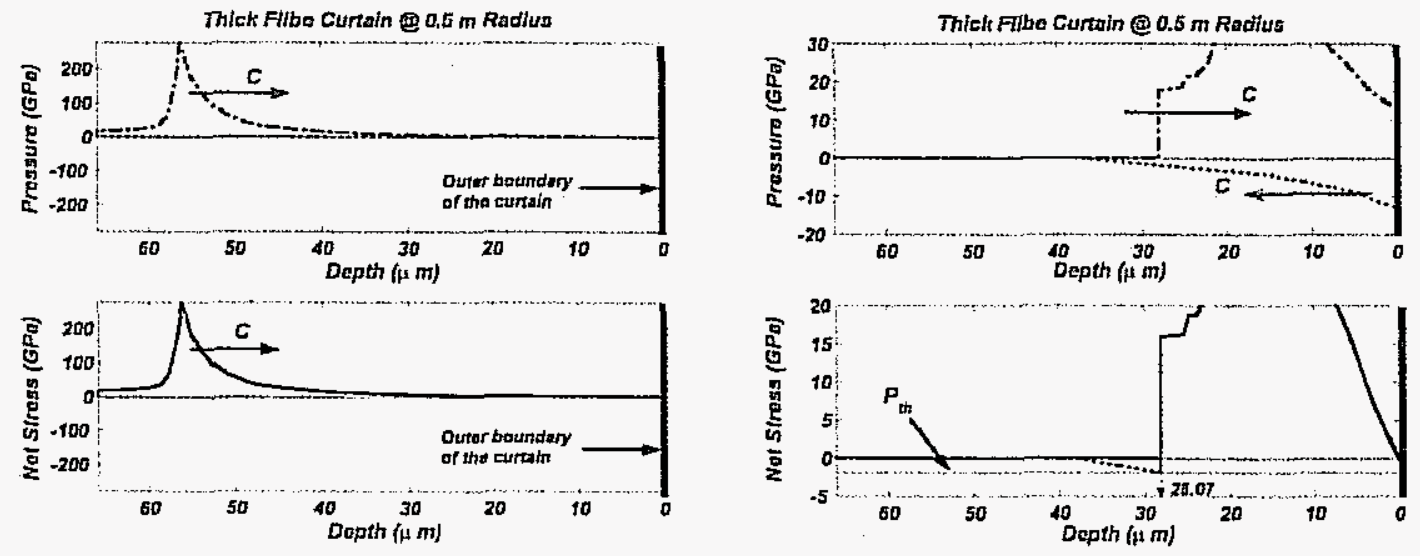

Figure 7 Illustration of spalling in a thick liquid wall at a radius of $0.5 \mathrm{~m}$ from the micro-explosion under the scaled initial pressure pulse shown in Fig. 6 (the shock is assumed to move at the speed of sound, C). The ordinate scale on the right-hand-side figures have been magnified for clarity. (Note that these results are only dependent on the distance from the back of the jet and not on the actual thickness of the jet). 


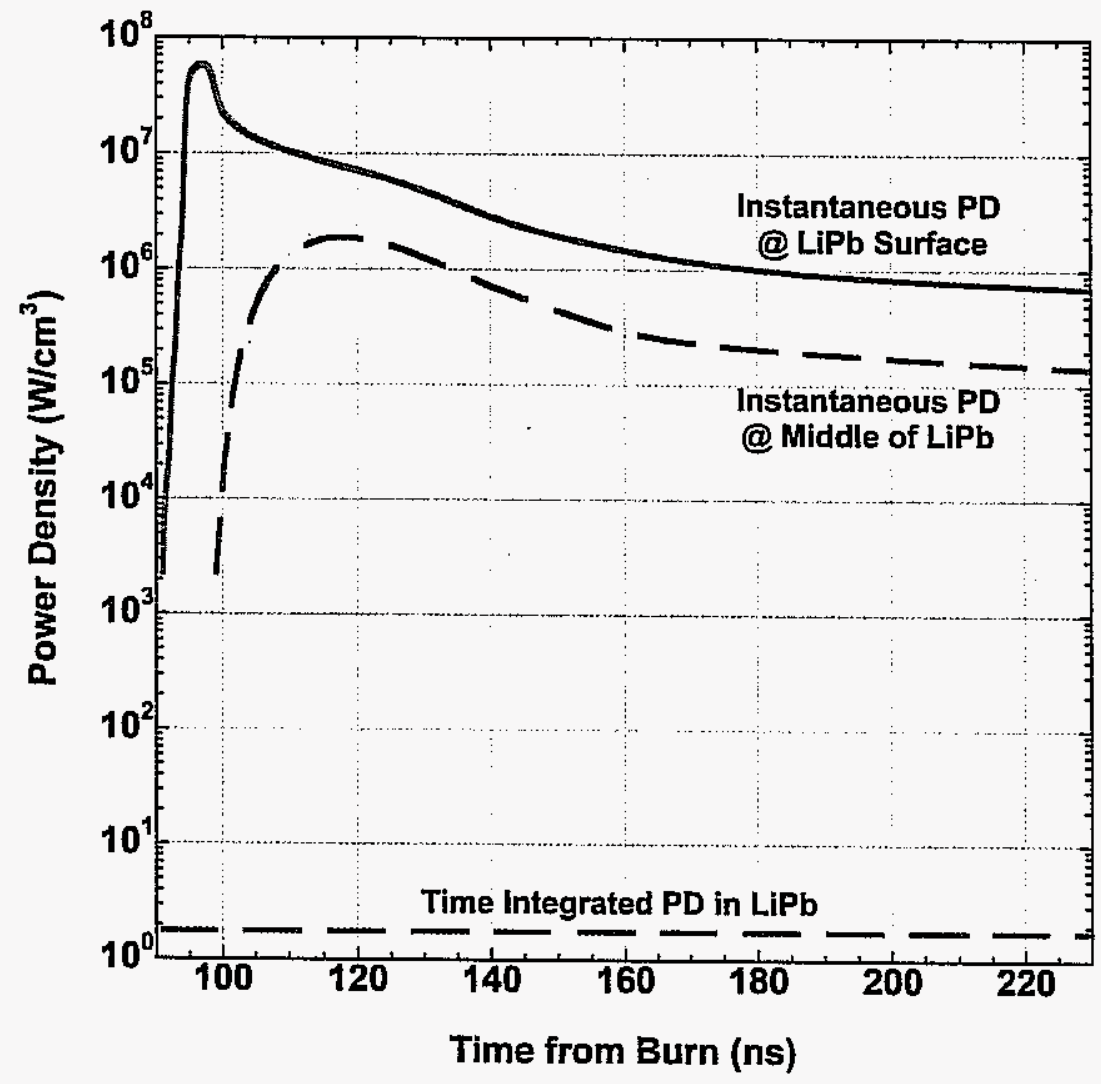

Fig. 8. Instantaneous nuclear energy deposition at the surface and middle of the $2 \mathrm{~m}$ thick $\mathrm{Pb}-17 \mathrm{Li}$ wall of the HIBAAL design. 


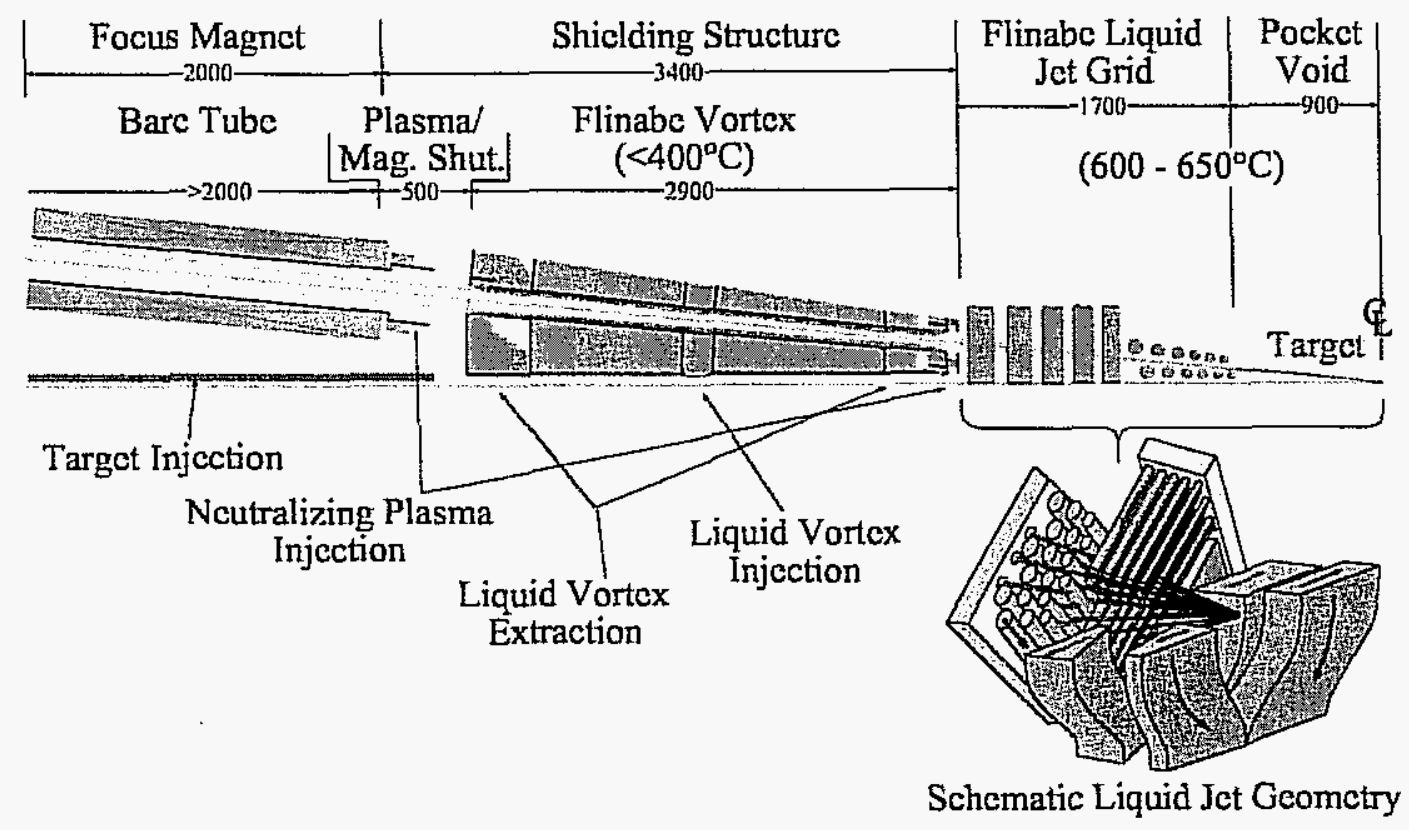

Figure 9 RPD beamline schematic $[22,25]$. 


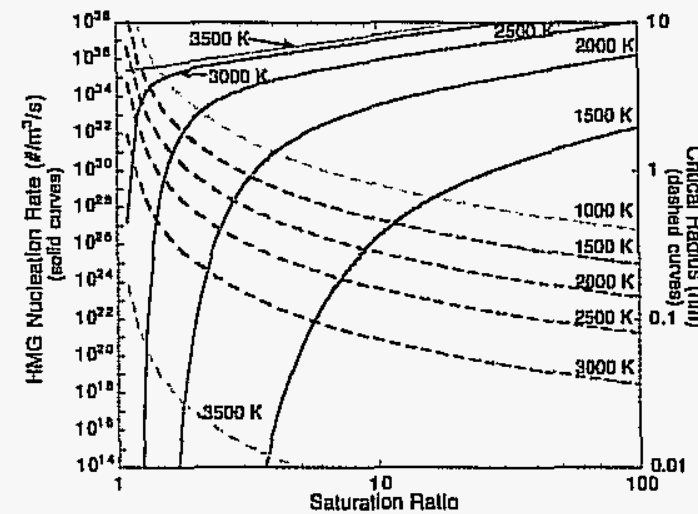

(a)

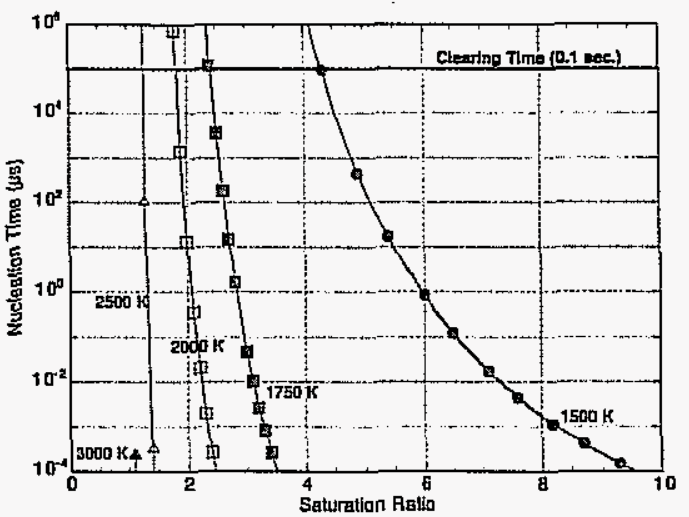

(b)

Figure 10. (a) Homogeneous nucleation rate (solid lines) and critical particle radii (dashed-lines) for nucleation of $\mathrm{Pb}$ aerosol particles. Note the highly non-linear behavior at moderate saturation ratios. (b) Illustration of the time required to nucleate $10^{15}$ particles $/ \mathrm{m}^{3}$. 


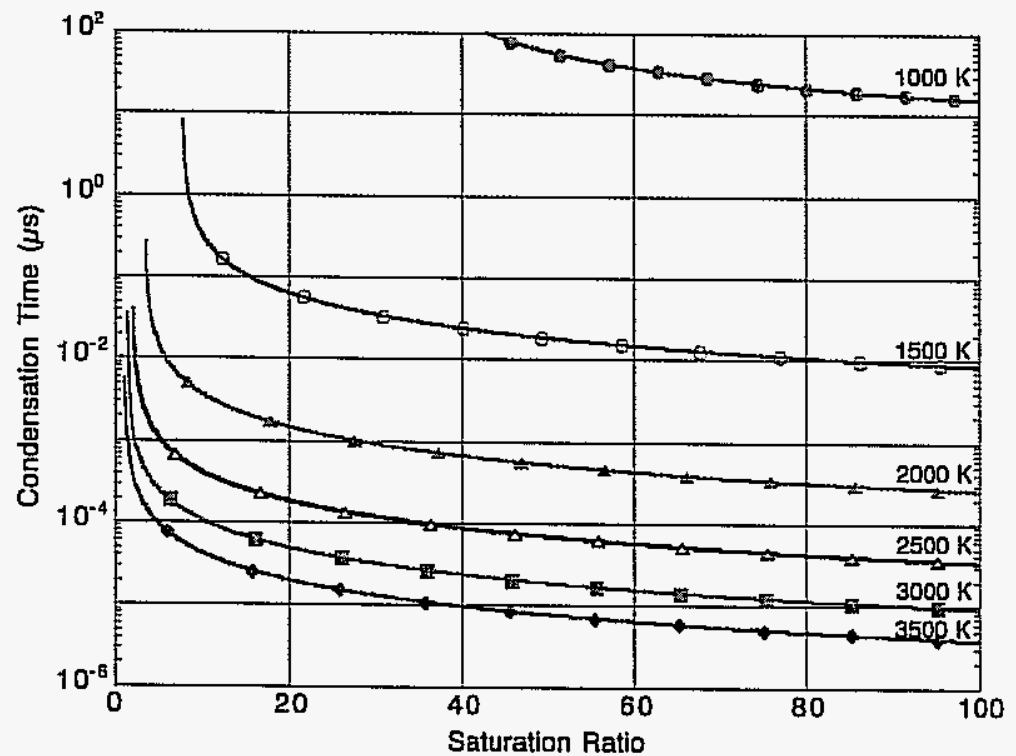

Figure 11. Time periods for condensation growth of a $1 \mu \mathrm{m} \mathrm{Pb}$ particle as a function of saturation ratio is less than $1 \mathrm{~ms}$. 


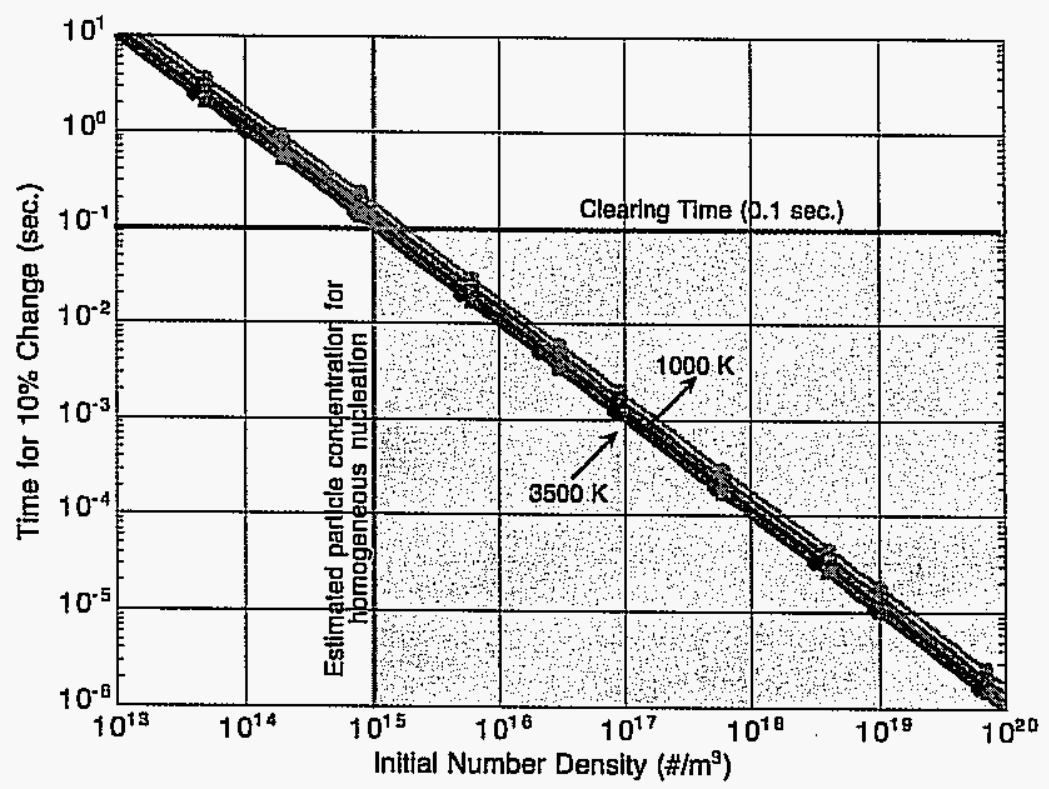

Figure 12. Coagulation changes the size distribution a number of $1 \mu \mathrm{m}$ aerosol particles in a time frame relevant to intermediate chamber clearing times. 


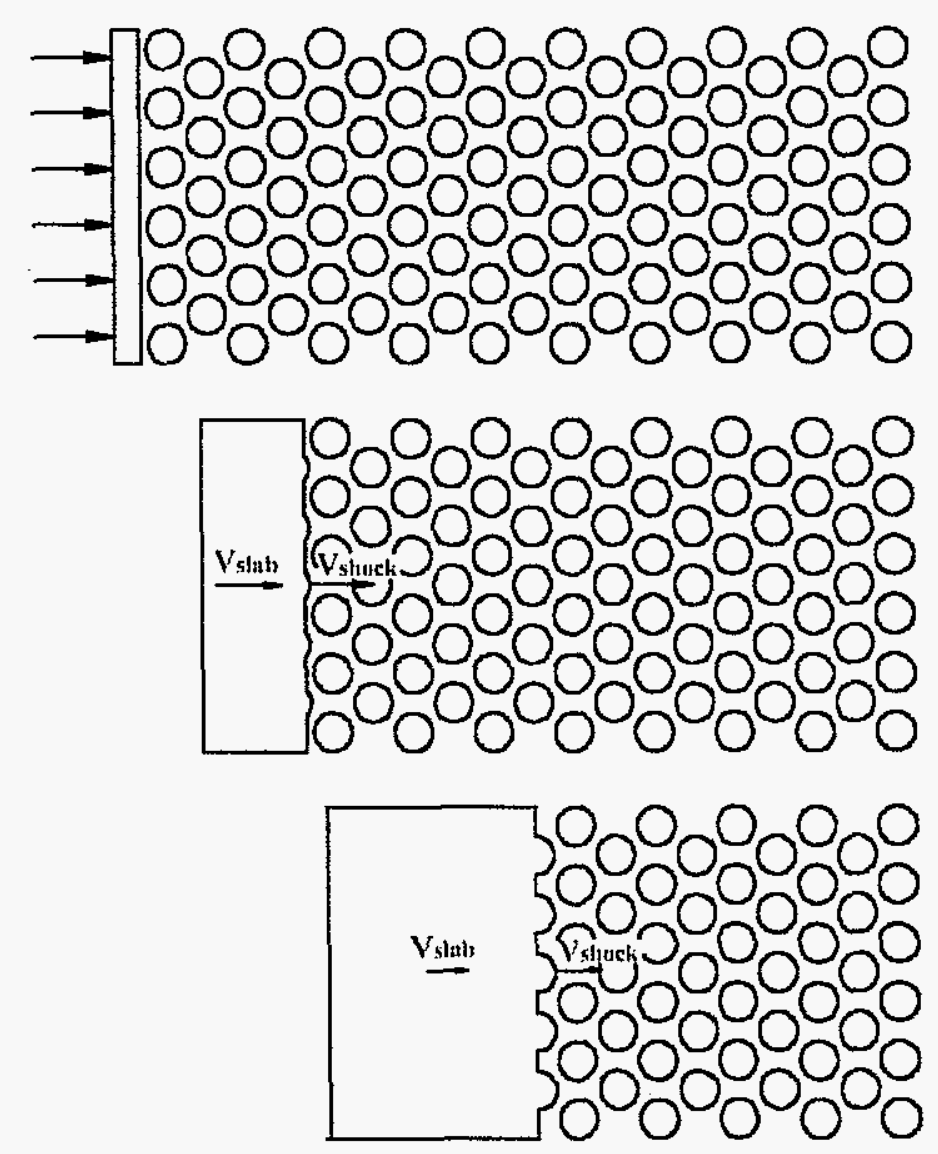

Figure 13 Schematic of voided slab jet compression. Note that the actual slab jets are each made of one sheet jet and an array of cylindrical jets [29]. 


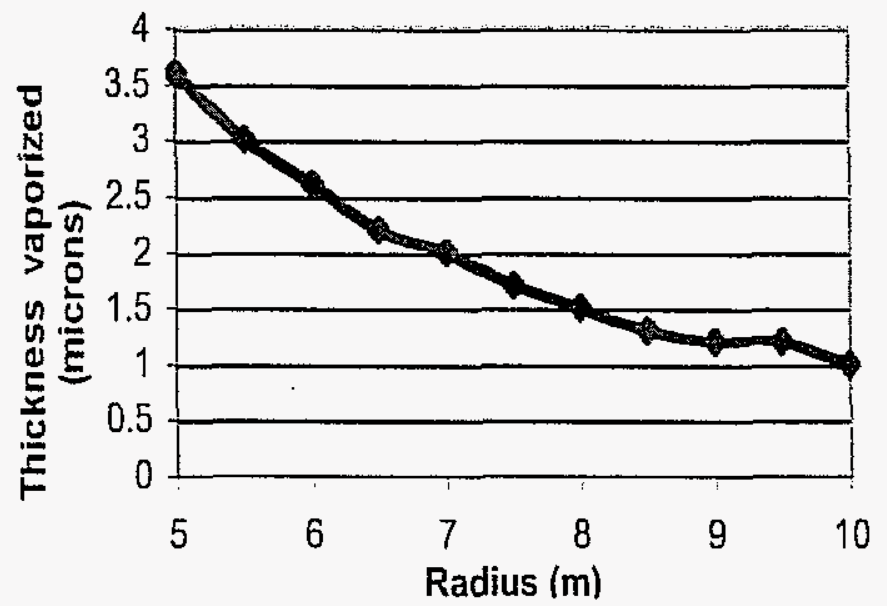

Figure 14. Vaporized flibe thickness for a thin film as a function of stand-off distance from target. 


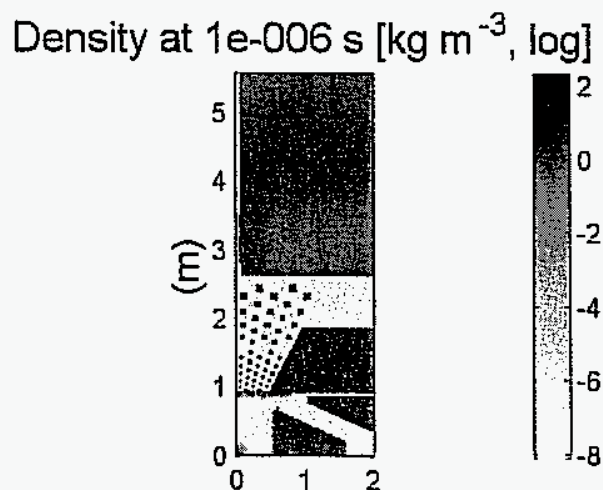

$(\mathrm{m})$
Density at $3 e-006 \mathrm{~s}\left[\mathrm{~kg} \mathrm{~m}^{-3}, \mathrm{log}\right]$

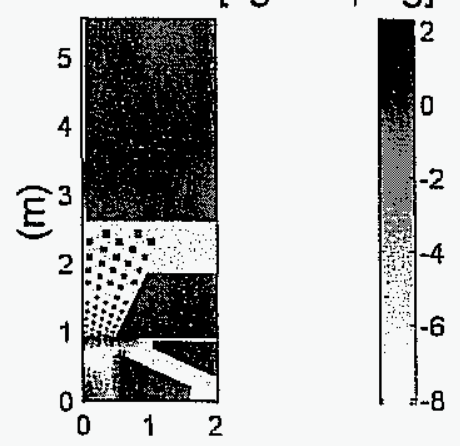

(m)

Density at $9 e-006 \mathrm{~s}\left[\mathrm{~kg} \mathrm{~m}^{-3}, \mathrm{log}\right] \quad$ Density at $2 \mathrm{e}-005 \mathrm{~s}\left[\mathrm{~kg} \mathrm{~m}^{-3}, \mathrm{log}\right]$

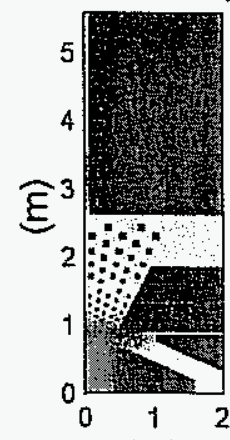

(m)
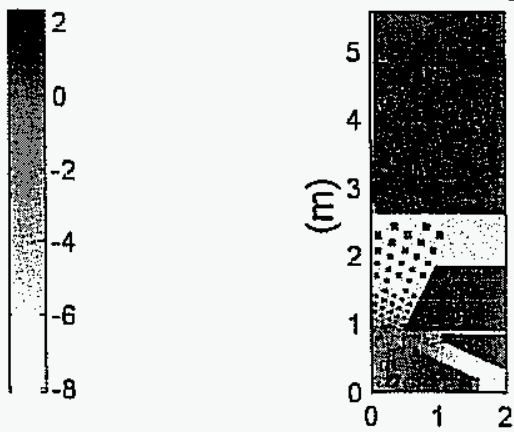

(m)

Figure 15 TSUNAMI density contour plots at various times. The density of the liquid and solid structures is arbitrary low. 


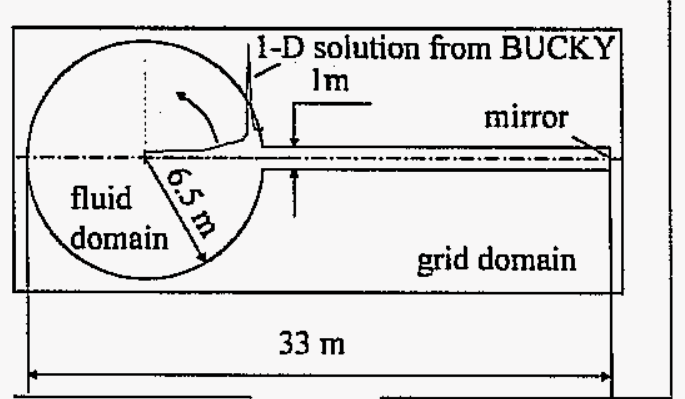

(a)

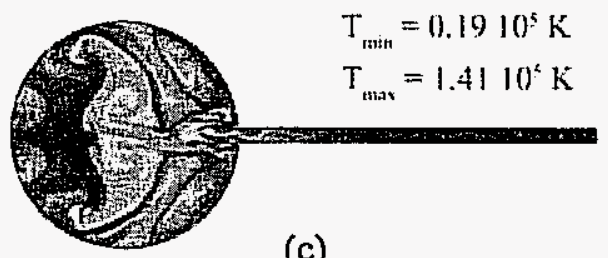

(c)

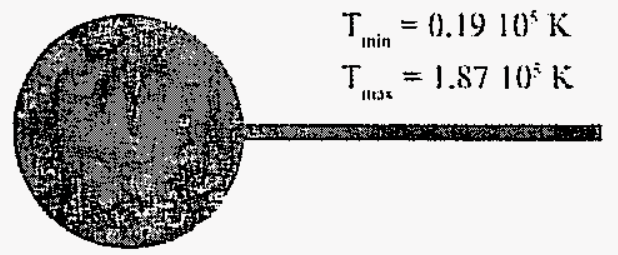

(b)

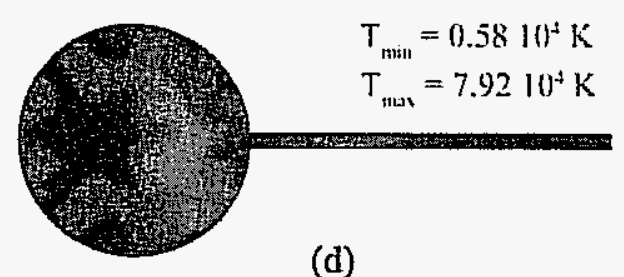

(d)

Figure 16 Example simulation of an IFE dry wall chamber with Xe as background gas. (a) Geometry of the chamber with the initial conditions imposed from a 1-D solution obtained by the BUCKY code. The temperature field, as shown in (b) through (d), is given $100 \mathrm{~ms}$ after the target implosion. Solution (b) is obtained by setting diffusive terms to zero. A temperature dependent viscosity is estimated by an empirical law in (c), while the conductivity is neglected. Case (d) features a similar empirical law for conductivity while the viscosity is set to zero. In all the cases the protective gas is Xenon. 


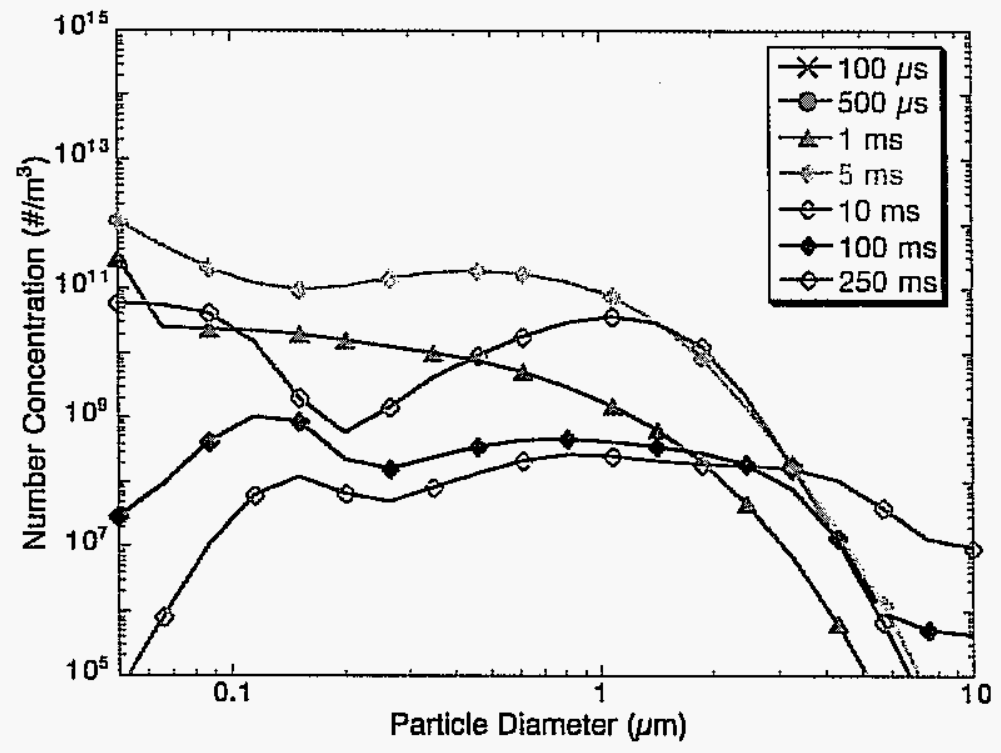

Figure 17. TOPGUN simulation results for aerosol production in a $6.5 \mathrm{~m}$ spherical chamber with a liquid lead wall indicate a significant population of moderately sized aerosol particles existing in the central region of the IFE chamber. 

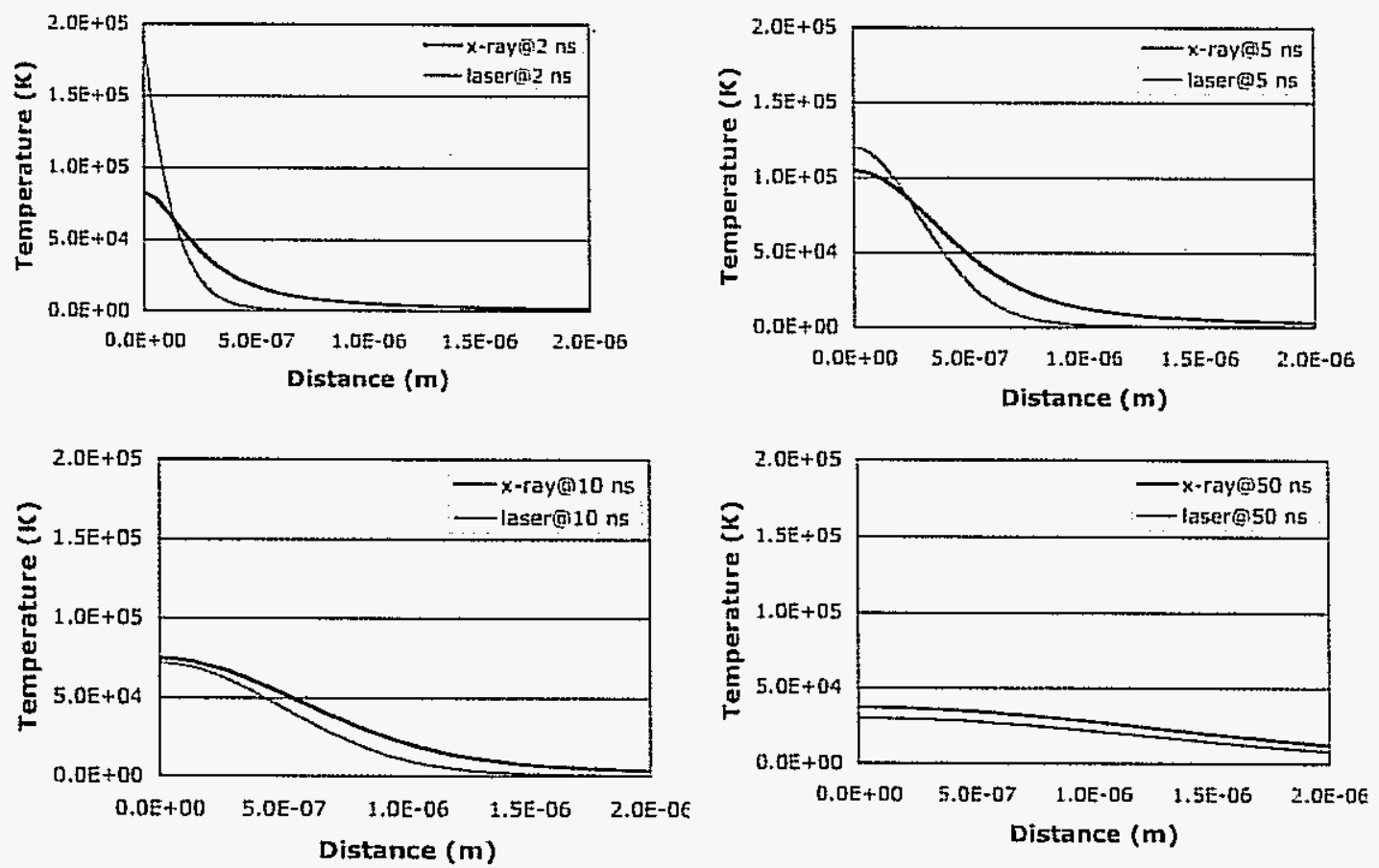

Figure 18 Thermal response of the surface of $\mathrm{Pb}$ exposed to a 2-ns laser pulse and a 2-ns soft X-ray pulse. The time following initiation of the energy source is $2 \mathrm{~ns}, 5 \mathrm{~ns}, 10 \mathrm{~ns}$ and $50 \mathrm{~ns}$. 


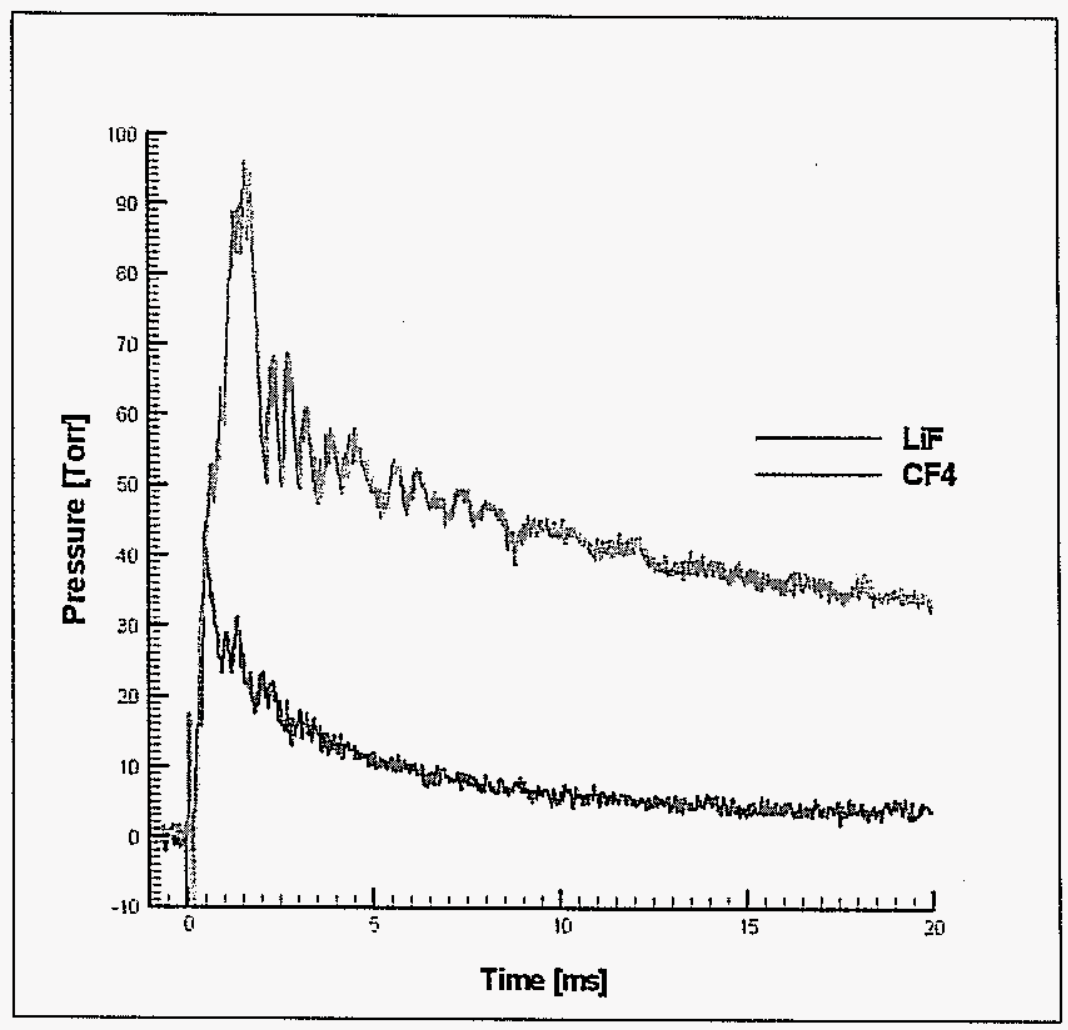

Figure 19. Pressure history of Teflon and LiF shots. 


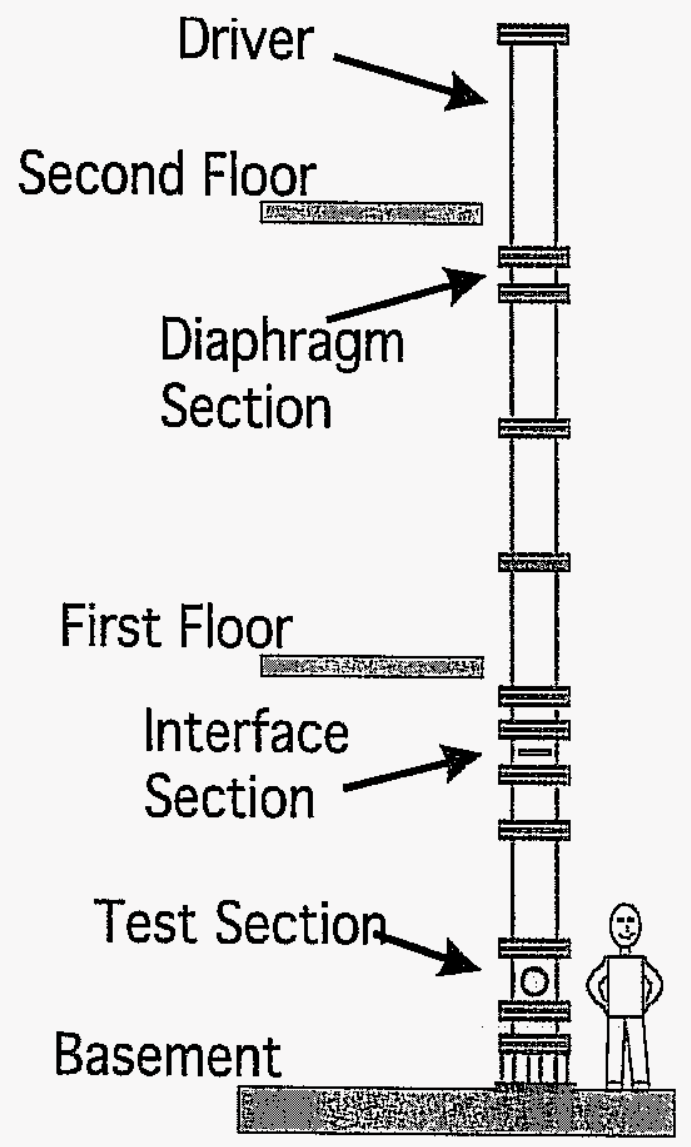

Figure 20 UW shock tube schematic. 


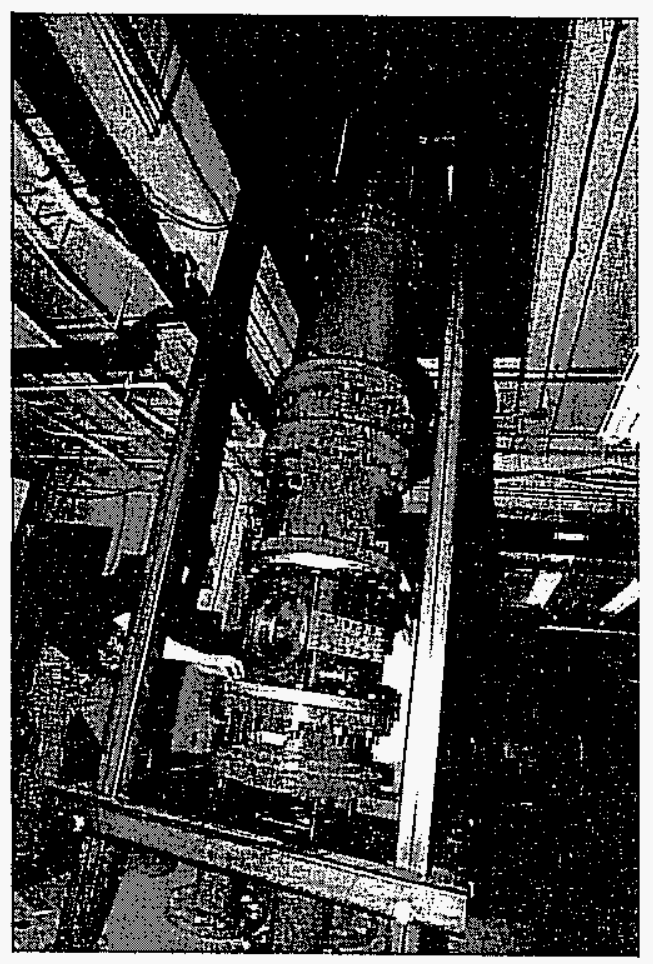

Figure 21 Image of UW shock tube facility's bottom third. 

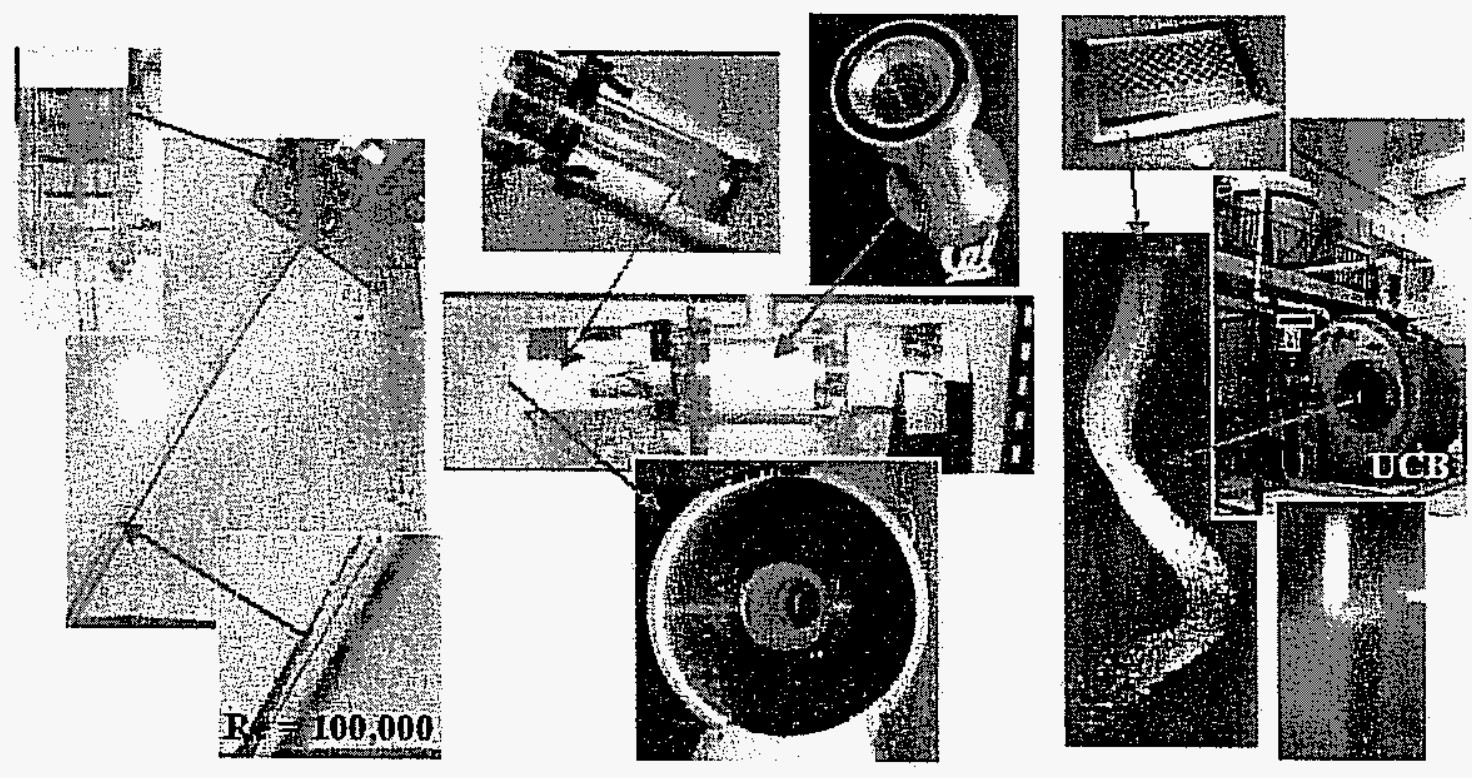

Fig. 22 Three of UCB jet experiments: high Reynolds number cylindrical jets, beamline vortex flow, and oscillating voided jets [28]. 


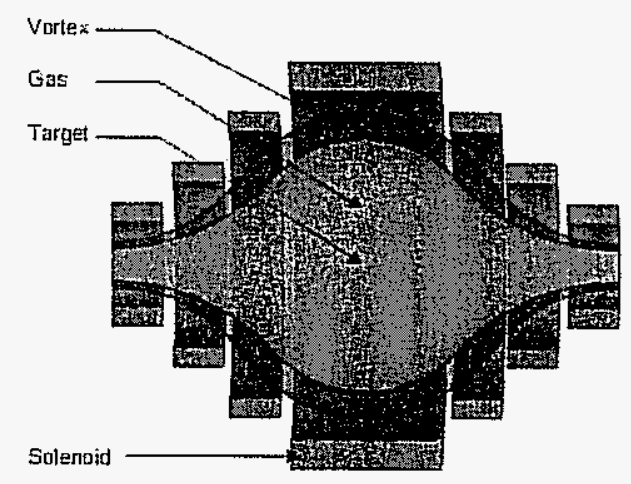

Figure 23 Schematic of the vortex chamber [43]. The first wall is not depicted. 


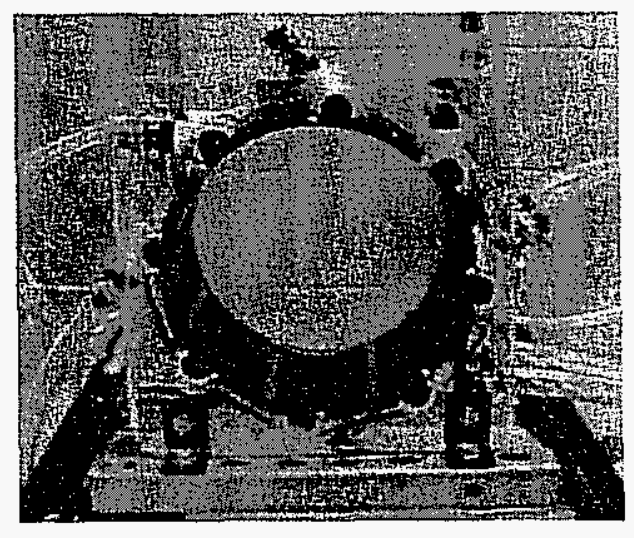

Figure 24 UCB large-vortex experimental setup [55]. 


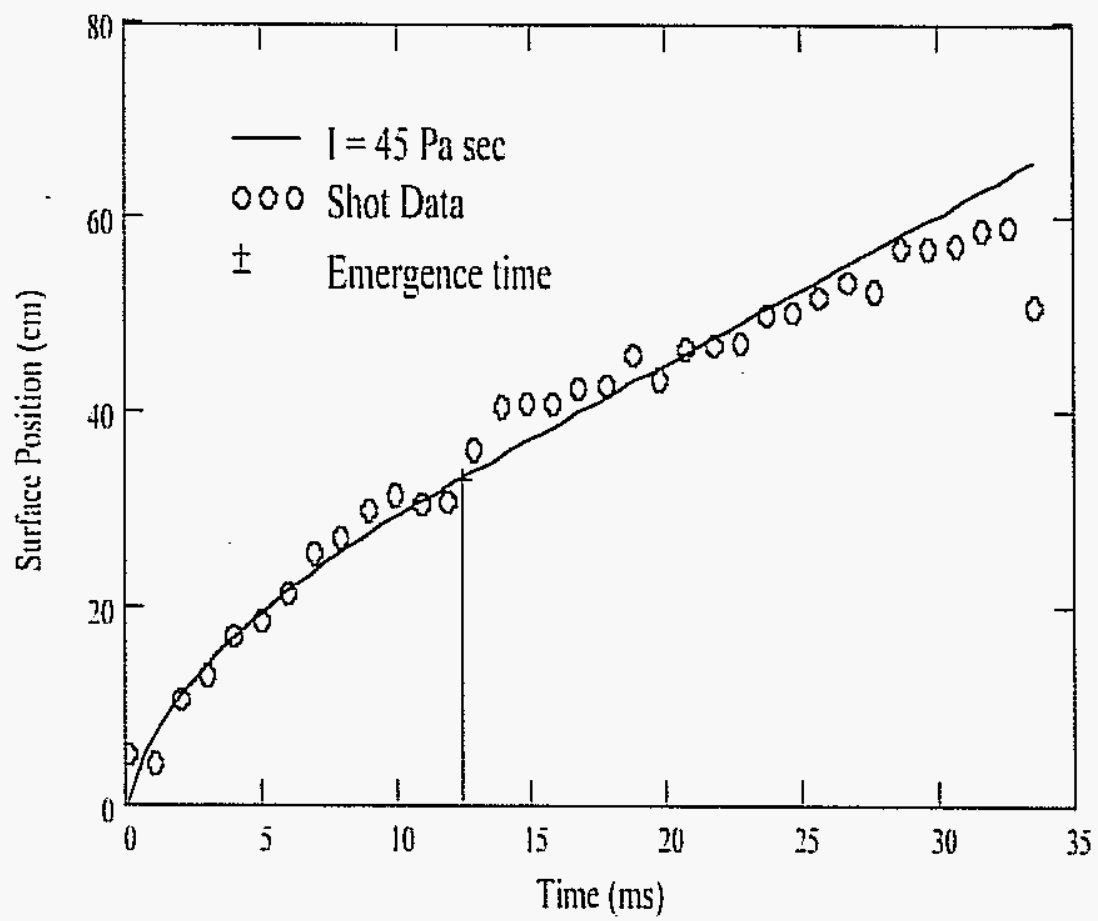

Figure 25 Comparison of snowplow compression model with experimental data (the position of the target-facing surface of the slab that makes up part of the oscillating voided jet is shown as a function of time) [29]. 


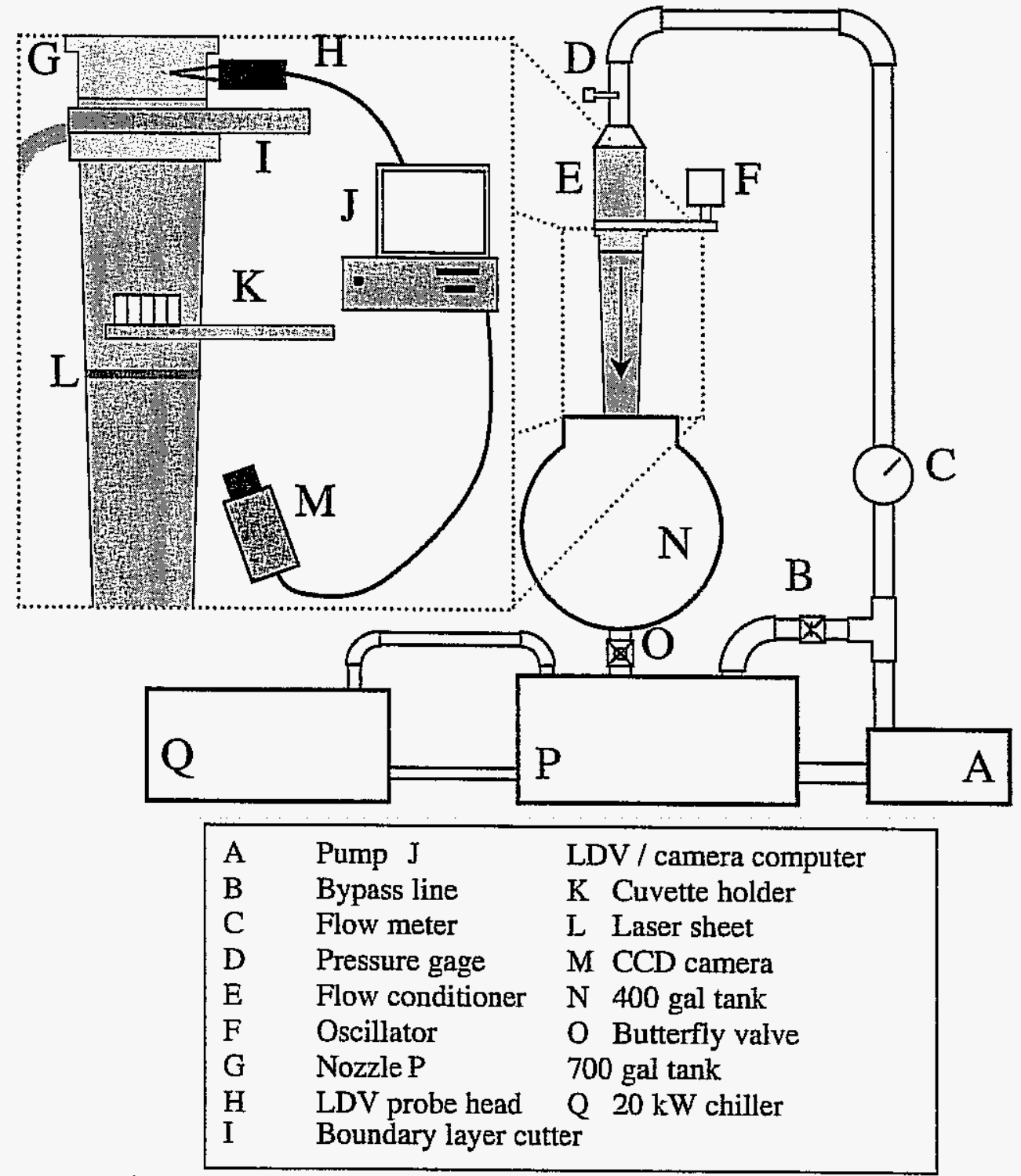

Figure 26 The Georgia Tech Large-Scale Hydraulic Test Facility. 